\title{
Non-isothermal flow of a thin film of fluid with temperature-dependent viscosity on a stationary horizontal cylinder
}

\author{
G. A. Leslie, S. K. Wilson* and B. R. Duffy ${ }^{\dagger}$ \\ Department of Mathematics and Statistics, \\ University of Strathclyde, 26 Richmond Street, \\ Glasgow G1 1XH, United Kingdom
}

(Dated: 30th September 2010, revised 2nd March and 2nd May 2011)

\begin{abstract}
A comprehensive description is obtained of the two-dimensional steady gravity-driven flow with prescribed volume flux of a thin film of Newtonian fluid with temperature-dependent viscosity on a stationary horizontal cylinder. When the cylinder is uniformly hotter than the surrounding atmosphere (positive thermoviscosity) the effect of increasing the heat transfer to the surrounding atmosphere at the free surface is to increase the average viscosity and hence reduce the average velocity within the film, with the net effect that the film thickness (and hence the total fluid load on the cylinder) is increased to maintain the fixed volume flux of fluid. When the cylinder is uniformly colder than the surrounding atmosphere (negative thermoviscosity) the opposite occurs. Increasing the heat transfer at the free surface from weak to strong changes the film thickness everywhere (and hence the load, but not the temperature or the velocity) by a constant factor which depends only on the specific viscosity model considered. The effect of increasing the thermoviscosity is always to increase the film thickness and hence the load. In the limit of strong positive thermoviscosity the velocity is small and uniform outside a narrow boundary layer near the cylinder leading to a large film thickness, while in the limit of strong negative thermoviscosity the velocity increases from zero at the cylinder to a large value at the free surface leading to a small film thickness.
\end{abstract}

\footnotetext{
* Author to whom correspondence should be addressed. Electronic mail: s.k.wilson@strath.ac.uk, Telephone: + 44 (0) 1415483820 , Fax: + 44 (0) 1415483345.

$\dagger$ Electronic mail: b.r.duffy@strath.ac.uk, Telephone: + 44 (0) 141548 3645, Fax: + 44 (0) 141548 3345.
} 


\section{INTRODUCTION}

The non-isothermal flow of a thin film of fluid on a heated or cooled horizontal circular cylinder is relevant to many industrial situations, including heat exchangers and various coating processes. Pioneering work on this problem was done by Nusselt ${ }^{1,2}$, who studied the steady condensation of a quiescent surrounding vapour (steam) into a thin film of fluid (water) on a stationary horizontal cylinder. Extensions of this basic problem have been considered by many subsequent authors, including, for example, Sparrow and $\mathrm{Gregg}^{3}$, Nicol et al. ${ }^{4}$ and Shu and Wilks ${ }^{5}$, who included fluid inertia and thermal advection in the film, Shekriladze and Gomelauri ${ }^{6}$, Fujii et al. ${ }^{7}$, Rose $^{8}$ and Chen and Lin $^{9}$, who considered the influence of flow of the vapour, and Sarma et al. ${ }^{10}$ and Yang and Lin $^{11}$, who considered turbulent flow in the film. Condensation onto an elliptical (rather than a circular) cylinder has been studied by Yang and $\mathrm{Hsu}^{12}$ for the case of laminar flow and by Lin and Yang ${ }^{13}$ for the case of turbulent flow. Another notable work on the non-isothermal flow of a thin film of fluid on a stationary horizontal cylinder is that by Reisfeld and Bankoff ${ }^{14}$, who undertook a pioneering investigation of unsteady flow on a heated or cooled cylinder due to gravity, surface tension, thermocapillary (i.e. variation of surface tension with temperature) and van der Waals forces. Subsequently, Conlisk and $\mathrm{Mao}^{15}$ investigated the unsteady flow of a thin film of fluid on a horizontal cylinder accounting for condensation from the surrounding vapour for both one-component and two-component fluids.

In a number of practical situations thermoviscosity (i.e. variation of viscosity with temperature) effects are significant, and as a result there have also been a number of studies of a variety of non-isothermal flows of fluids with temperature-dependent viscosities on a variety of substrates. In particular, Goussis and Kelly ${ }^{16,17}$ and Hwang and Weng ${ }^{18}$ investigated the stability of a layer of fluid with temperature-dependent viscosity flowing down a heated or cooled inclined substrate, while Reisfeld and Bankoff ${ }^{19}$ and $\mathrm{Wu}$ and Hwang ${ }^{20}$ 
independently considered the evolution and eventual rupture of a thin film of fluid with temperature-dependent viscosity on a heated horizontal substrate subject to surface tension and van der Waals forces. Selak and Lebon ${ }^{21}$ investigated the onset of convection in a quiescent layer of fluid with temperature-dependent viscosity on a heated or cooled horizontal substrate subject to both buoyancy and thermocapillary effects. Geophysical applications such as lava flows have motivated the study of the radial spreading of a thin film of fluid with temperature-dependent viscosity on a horizontal substrate by Bercovici ${ }^{22}$ who included thermal advection, and by Balmforth and Craster $^{23}$ who considered a viscoplastic fluid with a temperature-dependent viscosity and yield stress. Kabova and Kuznetsov ${ }^{24}$ calculated the steady flow of a thin film of fluid with temperature-dependent surface tension and viscosity down an inclined substrate. Wilson and Duffy ${ }^{25,26}$ and Duffy and Wilson ${ }^{27}$ studied the steady flow of a rivulet with temperature-dependent viscosity down a heated or cooled inclined substrate for three viscosity models (namely a linear, an exponential and an Eyring model). Sansom et al. ${ }^{28}$ considered the spreading of a thin film of fluid with temperaturedependent viscosity on a horizontal substrate for three viscosity models (namely a linear, an exponential and a biviscosity model) for both a heated or cooled substrate without internal heating within the film and for a substrate at the ambient temperature with constant internal heating within the film. Unsteady flow of a thin film of fluid with temperature-dependent surface tension and viscosity on a uniformly rotating disk was considered independently by Usha et al. ${ }^{29}$ and $\mathrm{Wu}^{30}$.

There is also a considerable body of literature on both two-dimensional and threedimensional isothermal thin-film flow on both the inside and the outside of a horizontal cylinder which is also of relevance here. Lin et al. ${ }^{31}$ investigated three-dimensional evolution and rupture of a film due to van der Waals forces. King et al. ${ }^{32}$ studied the three-dimensional evolution of a film on both a horizontal and an inclined cylinder. Band et al. ${ }^{33}$ considered two-dimensional flow driven by prescribed azimuthal variations in surface tension. Haimovich 
and Oron $^{34}$ investigated the effect of axial oscillations of the cylinder on the evolution and rupture of an axisymmetric film. In addition, there is a considerable and rapidly growing body of work on isothermal thin-film flow on both the inside (often called "rimming flow") and the outside (often called "coating flow") of a uniformly rotating circular cylinder building on the pioneering work by Moffatt ${ }^{35}$, Pukhnachev ${ }^{36}$ and Johnson ${ }^{37}$. For example, Duffy and Wilson ${ }^{38}$ considered steady "curtain" flows on the outside of both a stationary and a uniformly rotating cylinder, while Ashmore et al. ${ }^{39}$, Villegas-Díaz et al. ${ }^{40}$ and Benilov et al. ${ }^{41}$ investigated various aspects of rimming flow, and Evans et al. ${ }^{42}$, Kelmanson ${ }^{43}$ and Hunt ${ }^{44}$ investigated various aspects of coating flow (the latter in the case of an elliptical cylinder).

However, despite the practical importance of the problem surprisingly little work has been done on the steady gravity-driven flow of a thin film of fluid with temperature-dependent viscosity on a heated or cooled horizontal cylinder. Recently Duffy and Wilson ${ }^{45}$ examined this problem for both a stationary and a uniformly rotating cylinder in the special case when the free surface is at the same uniform temperature as the surrounding atmosphere (i.e. at leading order in the limit of large Biot number). In particular, they found that in this case the film thickness (and hence the load, but not the temperature or the velocity) can be obtained from that in the isothermal case by a simple re-scaling. However, they did not appreciate that other re-scalings are possible in the case of a stationary cylinder or undertake any analysis of the solution obtained. In the present work we build on the foundations laid by Duffy and Wilson ${ }^{45}$ to obtain a comprehensive description of the steady gravity-driven flow with prescribed volume flux of a thin film of fluid with temperaturedependent viscosity on a heated or cooled stationary horizontal cylinder. In particular, we investigate the effect of varying the heat transfer to or from the atmosphere at the free surface and the thermoviscosity. 


\section{THE VISCOSITY MODEL AND THE THERMOVISCOSITY NUMBER}

So far as possible we will present results for a general viscosity model $\mu=\mu(T)$, where $\mu(T)$ is a monotonically decreasing function of temperature $T$ satisfying (without loss of generality) $\mu=\mu_{0}$ and $\mathrm{d} \mu / \mathrm{d} T=-\lambda$ when $T=T_{0}$, where $\lambda>0$ is a prescribed positive constant and $T_{0}$ is the uniform temperature of the cylinder at which $\mu$ takes the constant value $\mu_{0}$. However, when it is necessary to specify a particular viscosity model and, in particular, for illustrative purposes, we adopt the widely used exponential viscosity model

$$
\mu(T)=\mu_{0} \exp \left(-\frac{\lambda\left(T-T_{0}\right)}{\mu_{0}}\right)
$$

(see, for example, Goussis and Kelly ${ }^{16,17}$, Hwang and Weng ${ }^{18}$, Selak and Lebon ${ }^{21}$, Balmforth and Craster $^{23}$, and Wilson and Duffy ${ }^{25}$ ).

Regardless of the specific viscosity model under consideration, an appropriate nondimensional measure of thermoviscosity (i.e. the variation of viscosity with temperature) is provided by the thermoviscosity number, $V$, defined by

$$
V=\frac{\lambda\left(T_{0}-T_{\infty}\right)}{\mu_{0}}
$$

where $T_{\infty}$ is the uniform temperature of the atmosphere. Since the thermoviscosity number has the same sign as $T_{0}-T_{\infty}$, situations in which the cylinder is hotter (colder) than the atmosphere correspond to positive (negative) values of $V$. In practice, the magnitude of $V$ can vary over several orders of magnitude from arbitrarily small values (when the viscosity is effectively independent of temperature and/or when the magnitude of the heating or cooling is small) to reasonably large values (when the viscosity is strongly dependent on temperature and/or when the magnitude of the heating or cooling is large). For example, using the parameter values given by Selak and Lebon ${ }^{21}$ in the case $\left|T_{0}-T_{\infty}\right|=25 \mathrm{~K}$ yields $|V|=0.3825$ for acetic acid, $|V|=0.5225$ for silicone oil, $|V|=0.625$ for water, and $|V|=2.5125$ for glycerol, while Balmforth and Craster ${ }^{23}$ give "typical" values of $|V|=1$ for 


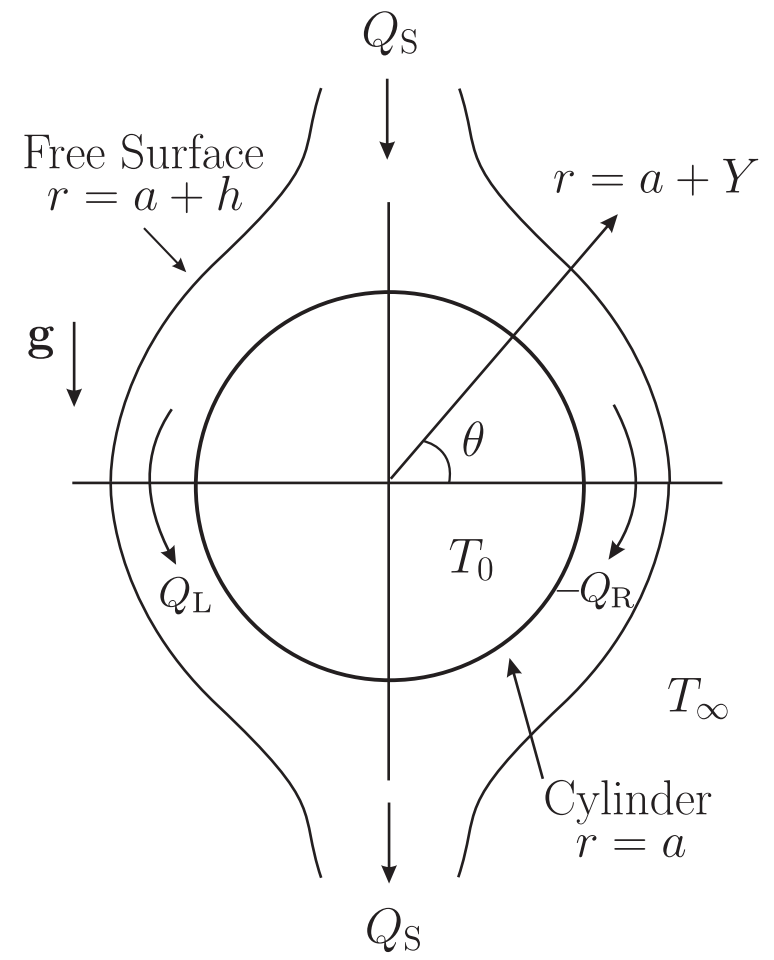

FIG. 1: Geometry of the problem: steady two-dimensional flow of a thin film of Newtonian fluid with temperature-dependent viscosity on a stationary horizontal cylinder which may be either uniformly hotter or colder than the surrounding atmosphere.

wax and slurry, $|V|=5$ for basaltic lava, $|V|=7$ for syrup, and $|V|=10-18$ for silicic lava. Hence we will consider the full range of values from $V=0$ to the limits $V \rightarrow \infty$ and $V \rightarrow-\infty$ in the present work.

\section{PROBLEM FORMULATION}

Consider two-dimensional steady gravity-driven flow of a thin film of Newtonian fluid with uniform density $\rho$ and temperature-dependent viscosity $\mu=\mu(T)$, where $T$ denotes the (in general) non-uniform temperature of the fluid, on a stationary circular cylinder of radius $a$ with its axis horizontal, the cylinder being at a uniform temperature $T_{0}$, which may be either hotter or colder than the uniform temperature $T_{\infty}\left(\neq T_{0}\right)$ of the surrounding atmosphere.

Referred to polar coordinates $r=a+Y$ (with origin at the cylinder's axis) and $\theta$ (measured counter-clockwise from the horizontal) as shown in Figure 1, we take the free surface of 
the fluid to be at $r=a+h$, the film thickness being denoted by $h$. The fluid velocity $\mathbf{u}=u \mathbf{e}_{\theta}+v \mathbf{e}_{r}$ (where $\mathbf{e}_{\theta}$ and $\mathbf{e}_{r}$ denote unit vectors in the azimuthal and radial directions, respectively), pressure $p$ and temperature $T$ are governed by the familiar mass-conservation, Navier-Stokes and energy equations. On the cylinder $r=a$ the velocity $\mathbf{u}$ satisfies the no-slip and no-penetration conditions, and the temperature is $T=T_{0}$ (a prescribed constant). On the free surface $r=a+h$ the usual normal and tangential stress balances and the kinematic condition apply, as does Newton's law of cooling

$$
-k_{\mathrm{th}} \nabla T \cdot \mathbf{n}=\alpha_{\mathrm{th}}\left(T-T_{\infty}\right)
$$

where $k_{\mathrm{th}}$ denotes the thermal conductivity of the fluid (assumed constant), $\alpha_{\mathrm{th}}(\geq 0)$ denotes an empirical surface heat-transfer coefficient, and $\mathbf{n}$ denotes the unit outward normal to the free surface. Surface tension, viscous dissipation, inertia and thermal advection are all neglected.

Since the flow is steady, the volume flux per unit axial length $Q$ (measured positive in the direction of increasing $\theta$ ) is a piecewise constant, and since (as we shall show) the film thickness $h$ always becomes unbounded at the top $(\theta=\pi / 2)$ and the bottom $(\theta=-\pi / 2)$ of the cylinder (where the tangential component of gravity is zero), it is natural to follow previous studies of the isothermal problem (see, for example, Duffy and Wilson ${ }^{38}$ ) and to interpret this as a curtain of fluid with prescribed constant volume flux $Q_{\mathrm{S}}(>0)$ falling onto the top of the cylinder and splitting into two films with constant azimuthal fluxes $Q=Q_{\mathrm{R}}$ and $Q=Q_{\mathrm{L}}$ round the right-hand and left-hand sides of the cylinder, respectively, with a corresponding curtain (also with flux $Q_{\mathrm{S}}$ ) falling off at the bottom of the cylinder. By global conservation of mass these fluxes are related by $Q_{\mathrm{S}}=Q_{\mathrm{L}}-Q_{\mathrm{R}}$, but the relative split of the flux between the two sides of the cylinder is not determined by the present theory. In particular, the flow need not necessarily have left-to-right symmetry (i.e. $Q_{\mathrm{R}}$ and $Q_{\mathrm{L}}$ need not necessarily be equal to $-Q_{\mathrm{S}} / 2$ and $Q_{\mathrm{S}} / 2$, respectively).

The total fluid load (i.e. the mass of fluid) per unit axial length on either side of the 
cylinder $M$ is denoted by $M=M_{\mathrm{R}}$ and $M=M_{\mathrm{L}}$ on the right-hand and left-hand sides of the cylinder, respectively, and hence the total fluid load on the cylinder is given by $M_{\mathrm{R}}+M_{\mathrm{L}}$.

We will consider only thin films, whose aspect ratio $\epsilon$, defined by

$$
\epsilon=\left(\frac{\mu_{0} Q_{\mathrm{S}}}{\rho g a^{3}}\right)^{1 / 3} \ll 1,
$$

is small. We non-dimensionalise and scale the system by writing

$$
\begin{gathered}
r=a\left(1+\epsilon Y^{*}\right), \quad h=\epsilon a h^{*}, \quad u=U u^{*}, \quad v=\epsilon U v^{*} \\
p=p_{\mathrm{a}}+\epsilon a \rho g p^{*}, \quad T=T_{\infty}+\left(T_{0}-T_{\infty}\right) T^{*}, \quad \mu=\mu_{0} \mu^{*} \\
Q=Q_{\mathrm{S}} Q^{*}=\epsilon a U Q^{*}, \quad Q_{\mathrm{R}}=Q_{\mathrm{S}} Q_{\mathrm{R}}^{*}=\epsilon a U Q_{\mathrm{R}}^{*}, \quad Q_{\mathrm{L}}=Q_{\mathrm{S}} Q_{\mathrm{L}}^{*}=\epsilon a U Q_{\mathrm{L}}^{*} \\
M=\epsilon \rho a^{2} M^{*}, \quad M_{\mathrm{R}}=\epsilon \rho a^{2} M_{\mathrm{R}}^{*}, \quad M_{\mathrm{L}}=\epsilon \rho a^{2} M_{\mathrm{L}}^{*}
\end{gathered}
$$

where the characteristic azimuthal fluid velocity $U$, defined to be equal to $Q_{\mathrm{S}} / \epsilon a$, is given by

$$
U=\left(\frac{\rho g Q_{\mathrm{S}}^{2}}{\mu_{0}}\right)^{1 / 3}
$$

and $p_{\mathrm{a}}$ is the constant pressure in the surrounding atmosphere. Note that the nondimensionalisation of temperature given in (5) incorporates the factor $T_{0}-T_{\infty}$, which can be either positive or negative, and so a little care is required in interpreting results for the non-dimensional temperature $T^{*}$ in terms of the dimensional temperature $T$. For clarity the star superscripts on non-dimensional variables will be omitted henceforth.

Expressed in non-dimensional variables the fluid occupies $0 \leq Y \leq h$ for $-\pi<\theta \leq \pi$, the flux $Q$ takes the values $Q=Q_{\mathrm{R}}$ on the right-hand side of the cylinder $|\theta|<\pi / 2$ and $Q=Q_{\mathrm{L}}$ on the left-hand side of the cylinder $\pi / 2<|\theta| \leq \pi$, with $Q_{\mathrm{L}}-Q_{\mathrm{R}}=1$; also the general viscosity model $\mu=\mu(T)$ satisfies $\mu=1$ and $\mathrm{d} \mu / \mathrm{d} T=-V$ when $T=1$, and, in particular, the exponential viscosity model (1) is given by

$$
\mu=\exp (-V(T-1))
$$

At leading order in $\epsilon$ the governing equations become

$$
u_{\theta}+v_{Y}=0, \quad\left(\mu u_{Y}\right)_{Y}=\cos \theta, \quad p_{Y}=-\sin \theta, \quad T_{Y Y}=0
$$


together with the boundary conditions

$$
\begin{gathered}
u=0, \quad v=0 \text { and } T=1 \quad \text { on } Y=0, \\
u_{Y}=0, \quad p=0 \text { and } T_{Y}+B T=0 \quad \text { on } \quad Y=h,
\end{gathered}
$$

where $B=\epsilon a \alpha_{\mathrm{th}} / k_{\mathrm{th}}(\geq 0)$ is the non-dimensional Biot number (a non-dimensional measure of heat transfer to or from the atmosphere at the free surface) and the suffixes $Y$ and $\theta$ denote the appropriate partial derivatives. The special case $B=0$ corresponds to that of a perfectly insulated free surface with no heat transfer (i.e. $T_{Y}=0$ at $Y=h$ ), while at leading order in the limit $B \rightarrow \infty$ the free surface is at the same uniform temperature as the atmosphere (i.e. $T=0$ at $Y=h$ ), and so we will consider the full range of values from $B=0$ to the limit $B \rightarrow \infty$ in the present work.

Introducing the rescaled variable $y=Y / h$ (so that the fluid occupies $0 \leq y \leq 1$ ) and solving (8) subject to (9) and (10) for the temperature $T=T(y, \theta)$, the azimuthal velocity $u=u(y, \theta)$ and the pressure $p=p(y, \theta)$ yields

$$
\begin{gathered}
T(y, \theta)=1-\frac{B h y}{1+B h}, \\
u(y, \theta)=-h^{2} \cos \theta \int_{0}^{y} \frac{1-\tilde{y}}{\mu(T(\tilde{y}, \theta))} \mathrm{d} \tilde{y}
\end{gathered}
$$

and

$$
p(y, \theta)=h(1-y) \sin \theta .
$$

The stream function $\psi=\psi(y, \theta)$ (non-dimensionalised with $Q_{\mathrm{S}}$ and satisfying $h u=\psi_{y}$ and $v=-\psi_{\theta}$ with $\psi=0$ on $\left.y=0\right)$ is given by

$$
\psi=-h^{3} \cos \theta \int_{0}^{y} \int_{0}^{\bar{y}} \frac{1-\tilde{y}}{\mu(T(\tilde{y}, \theta))} \mathrm{d} \tilde{y} \mathrm{~d} \bar{y}=-h^{3} \cos \theta \int_{0}^{y} \frac{(1-\tilde{y})(y-\tilde{y})}{\mu(T(\tilde{y}, \theta))} \mathrm{d} \tilde{y} .
$$

The volume flux $Q(=\psi(1, \theta))$ is given by

$$
Q=h \int_{0}^{1} u \mathrm{~d} y=-h^{3} \cos \theta \int_{0}^{1} \int_{0}^{y} \frac{1-\tilde{y}}{\mu(T(\tilde{y}, \theta))} \mathrm{d} \tilde{y} \mathrm{~d} y
$$


leading to

$$
Q=-\frac{h^{3} \cos \theta}{3} f
$$

where $f=f(\theta)(>0)$ is the fluidity of the fluid film, defined by

$$
f=3 \int_{0}^{1} \int_{0}^{y} \frac{1-\tilde{y}}{\mu(T(\tilde{y}, \theta))} \mathrm{d} \tilde{y} \mathrm{~d} y=3 \int_{0}^{1} \frac{(1-y)^{2}}{\mu(T(y, \theta))} \mathrm{d} y .
$$

In the special case of constant viscosity $\mu \equiv 1$ the fluidity is simply equal to unity, i.e. $f \equiv 1$. Note that, since the flux $Q$ is prescribed, (16) is the key equation which determines the film thickness $h$. Furthermore, since by definition $f>0$ and $h>0,(16)$ shows that $-Q / \cos \theta>0$, i.e. that $Q$ must always have the same $\operatorname{sign}$ as $-\cos \theta$. Thus we deduce that $-1<Q_{\mathrm{R}}<0$ and $0<Q_{\mathrm{L}}<1$, where the sign difference between $Q_{\mathrm{R}}$ and $Q_{\mathrm{L}}$ arises because the flux is everywhere downwards, and so it is in the direction of increasing $\theta$ on the left-hand side of the cylinder but is in the direction of decreasing $\theta$ on the right-hand side of the cylinder. In fact, the present analysis also applies to the flow on the left-hand side of the cylinder in the case $Q_{\mathrm{R}}=0, Q_{\mathrm{L}}=1$ (in which there is no fluid on the right-hand side of the cylinder), and to the flow on the right-hand side of the cylinder in the case $Q_{\mathrm{R}}=-1$, $Q_{\mathrm{L}}=0$ (in which there is no fluid on the left-hand side of the cylinder).

The fluid loads on the right-hand and the left-hand sides of the cylinder are given by

$$
M_{\mathrm{R}}=\int_{-\pi / 2}^{\pi / 2} h \mathrm{~d} \theta
$$

and

$$
M_{\mathrm{L}}=\int_{\pi / 2}^{\pi} h \mathrm{~d} \theta+\int_{-\pi}^{-\pi / 2} h \mathrm{~d} \theta
$$

respectively.

Thus, for a specific choice of viscosity model $\mu=\mu(T)$, the film thickness $h$ is determined in terms of $Q=Q_{\mathrm{R}}\left(-1 \leq Q_{\mathrm{R}}<0\right)$ on the right-hand side of the cylinder and in terms of $Q=Q_{\mathrm{L}}\left(0<Q_{\mathrm{L}} \leq 1\right)$ on the left-hand side of the cylinder by the algebraic equation (16) in which $f$ is given by (17), and the solutions for $T, u, p, M_{\mathrm{R}}$ and $M_{\mathrm{L}}$ are given explicitly by (11)-(13), (18) and (19), respectively. 
Note that while the present problem has been obtained as the leading-order approximation to the steady flow of a thin film of fluid on a large horizontal circular cylinder, exactly the same problem also describes the leading-order approximation to the steady flow of a thin film of fluid down any sufficiently slowly varying substrate with local angle of inclination to the horizontal $\alpha=\pi / 2-\theta$, where $0 \leq \alpha \leq \pi$. In particular, the present analysis applies to the widely studied problem of rectilinear flow down a planar substrate inclined at an angle $\alpha$ to the horizontal.

From (11), (12), (14), (16) and (17) it is clear that the Biot number $B$ appears only in the combinations $B h, B^{2} u, B^{3} \psi$ and $B^{3} Q$, and that $B h$ is a function of $-B^{3} Q / \cos \theta(>0)$. Thus, in particular, we could remove $B$ explicitly from the mathematical problem by rescaling $h$, $u, \psi$ and $Q$ appropriately; however, since this obscures the physical interpretation of the results obtained we retain $B$ explicitly in what follows.

Combining (11), (12), (14), (16) and (17) shows that $h, T, u, \psi$ and $f$ depend on $\theta$ only through $\cos \theta$, and so the flow has top-to-bottom symmetry, but (as we have already mentioned) not necessarily left-to-right symmetry.

Using (11) and (17) one may show that

$$
\frac{\mathrm{d}\left(f h^{3}\right)}{\mathrm{d} h}=\frac{3 h^{2}}{1+B h} \int_{0}^{1} \frac{(1-y)[2+3 B h(1-y)]}{\mu(T(y, \theta))} \mathrm{d} y>0
$$

and hence from (16) we find that $\partial h / \partial Q$ has the same sign as $Q$, which means that the film thickness at each station $\theta$ increases monotonically with $|Q|$.

Similarly, from (16) we find that $\mathrm{d} h / \mathrm{d} \theta$ has the same $\operatorname{sign}$ as $\tan \theta$, which means that the film thickness on the right-hand (left-hand) side of the cylinder increases monotonically away from its minimum value at $\theta=0(\theta=\pi)$.

Near the top and the bottom of the cylinder we have $h \rightarrow \infty, T \sim 1-y$, and $f \rightarrow \hat{f}$ as $\theta \rightarrow \pm \pi / 2$, where from (17) the constant $\hat{f}(>0)$, which depends only on the specific 
viscosity model considered, is defined by

$$
\hat{f}=3 \int_{0}^{1} \frac{T^{2}}{\mu(T)} \mathrm{d} T
$$

Specifically, from (16) the thin-film approximation ultimately fails as the film thickness becomes unbounded according to

$$
h=\left(\frac{3 Q}{(|\theta|-\pi / 2) \hat{f}}\right)^{1 / 3}-\frac{\hat{f}-\hat{g}}{\hat{f} B}+O\left(|\theta|-\frac{\pi}{2}\right)^{1 / 3} \quad \text { as } \quad \theta \rightarrow \pm \frac{\pi}{2},
$$

where the constant $\hat{g}(>0)$, which (like $\hat{f}$ ) also depends only on the specific viscosity model considered, is defined by

$$
\hat{g}=2 \int_{0}^{1} \frac{T}{\mu(T)} \mathrm{d} T
$$

Hereafter we will, for simplicity, restrict our attention to the flow on the right-hand side of the cylinder $(|\theta|<\pi / 2)$ with flux $Q=Q_{\mathrm{R}}\left(-1 \leq Q_{\mathrm{R}}<0\right)$ and load $M=M_{\mathrm{R}}$, from which the corresponding results for the flow on the left-hand side of the cylinder $(\pi / 2<|\theta|<\pi)$ with flux $Q=Q_{\mathrm{L}}\left(0<Q_{\mathrm{L}} \leq 1\right)$ and load $M=M_{\mathrm{L}}$ can be readily obtained.

\section{SPECIAL CASE OF CONSTANT VISCOSITY}

If either there is no heat transfer to or from the atmosphere at the free surface (i.e. in dimensional terms if $\alpha_{\mathrm{th}}=0$ ) so that $B=0$ (in which case the fluid film is isothermal with constant temperature $T \equiv 1$ ) or the viscosity is independent of temperature (i.e. in dimensional terms if $\lambda=0$ ) so that $V=0$ (in which case the fluid film is non-isothermal with non-constant temperature $T \not \equiv 1$ ), then the fluid has constant viscosity $\mu \equiv 1$ and fluidity $f \equiv 1$. In either case we recover the classical isothermal solution in which $h=h_{0}$, $u=u_{0}$ and $\psi=\psi_{0}$, where

$$
\begin{gathered}
h_{0}=\left(-\frac{3 Q}{\cos \theta}\right)^{1 / 3}, \\
u_{0}=-\frac{h_{0}^{2} \cos \theta}{2}(2-y) y
\end{gathered}
$$


and

$$
\psi_{0}=-\frac{h_{0}^{3} \cos \theta}{6}(3-y) y^{2}
$$

In particular, (24) shows that the film thickness $h_{0}$ increases monotonically with $|\theta|$ away from its minimum value of $(3|Q|)^{1 / 3}$ at $\theta=0$, becoming unbounded at the top and the bottom of the cylinder according to

$$
h_{0}=\left(\frac{3 Q}{|\theta|-\pi / 2}\right)^{1 / 3}+O\left(|\theta|-\frac{\pi}{2}\right)^{5 / 3} \quad \text { as } \quad \theta \rightarrow \pm \frac{\pi}{2},
$$

in agreement with the corresponding general results obtained in Section III. The load $M=$ $M_{0}$ is given by

$$
M_{0}=2 \int_{0}^{\pi / 2} h_{0} \mathrm{~d} \theta=C_{0}|Q|^{1 / 3}
$$

in which the numerical coefficient $C_{0}$ is given by

$$
C_{0}=2 \int_{0}^{\pi / 2}\left(\frac{3}{\cos \theta}\right)^{1 / 3} \mathrm{~d} \theta=\frac{2^{5 / 3} \pi^{2}}{3^{2 / 3} \Gamma\left(\frac{2}{3}\right)^{3}} \simeq 6.0669 .
$$

\section{GENERAL CASE OF NON-CONSTANT VISCOSITY}

In general, if there is heat transfer to or from the atmosphere at the free surface (i.e. in dimensional terms if $\alpha_{\text {th }}>0$ ) so that $B>0$ and the viscosity depends on temperature (i.e. in dimensional terms if $\lambda>0$ ) so that $V \neq 0$, then the fluid film is non-isothermal with, in general, non-constant temperature, viscosity and fluidity. In the particular case of the exponential viscosity model (7) we have

$$
\mu=\exp (-V(T-1))=\exp \left(\frac{B V h y}{1+B h}\right)=\exp (\mathcal{V} y)
$$

where, for brevity, we have introduced the notation $\mathcal{V}=\mathcal{V}(\theta)$ defined by

$$
\mathcal{V}=\frac{B V h}{1+B h}
$$

so that (12) yields the azimuthal velocity

$$
u=-\frac{h^{2} \cos \theta}{\mathcal{V}^{2}}[\mathcal{V}-1+(1-\mathcal{V}(1-y)) \exp (-\mathcal{V} y)]
$$




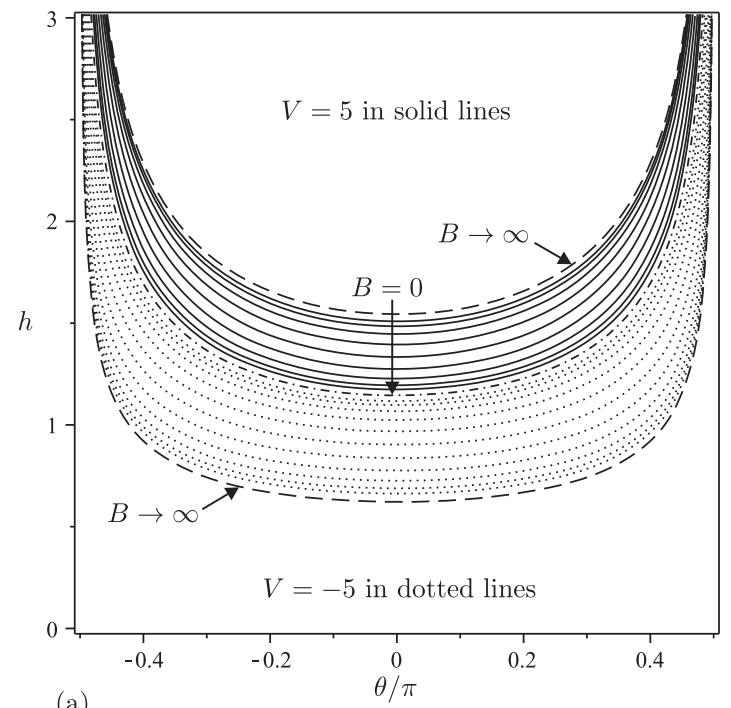

(a)

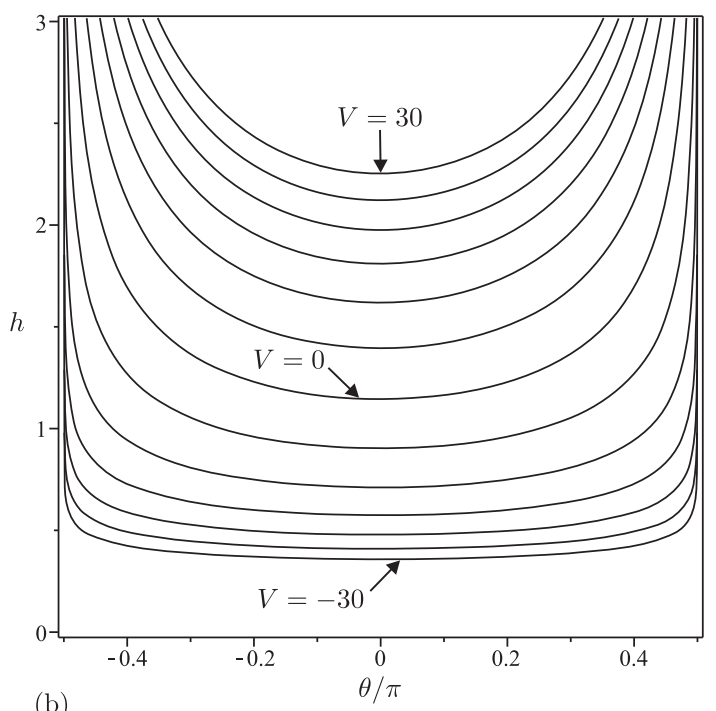

(b)

FIG. 2: Film thickness $h$ plotted as a function of $\theta / \pi$ for (a) $B=0$ (dash-dotted line), $B=10^{n}$ $(n=-1.25,-1,-0.75, \ldots, 1.25)$ in the case $V=-5$ (dotted lines) and $B=10^{n}(n=-1.25,-1,-0.75$, $\ldots, 0.75)$ in the case $V=5$ (solid lines) together with the leading order asymptotic solutions in the limit $B \rightarrow \infty$ in the cases $V=-5$ and $V=5$ (dashed lines), and for (b) $V=-30,-25,-20, \ldots, 30$ in the case $B=1$, when $Q=-1 / 2$.

(14) yields the stream function

$$
\psi=-\frac{h^{3} \cos \theta}{\mathcal{V}^{3}}[(\mathcal{V}-1)(\mathcal{V} y-1)+1-(2-\mathcal{V}(1-y)) \exp (-\mathcal{V} y)]
$$

and (17) yields the fluidity

$$
f=\frac{3}{\mathcal{V}^{3}}\left[(\mathcal{V}-1)^{2}+1-2 \exp (-\mathcal{V})\right]
$$

Note that $f$ is a monotonically decreasing function of $\mathcal{V}$ satisfying

$$
\begin{gathered}
f \sim \frac{6 \exp (-\mathcal{V})}{(-\mathcal{V})^{3}} \rightarrow \infty \quad \text { as } \quad \mathcal{V} \rightarrow-\infty \\
f=1-\frac{\mathcal{V}}{4}+O\left(\mathcal{V}^{2}\right) \text { as } \quad \mathcal{V} \rightarrow 0
\end{gathered}
$$

and

$$
f \sim \frac{3}{\mathcal{V}} \rightarrow 0 \quad \text { as } \quad \mathcal{V} \rightarrow \infty
$$

Figures 2 and 3 show the film thickness $h$ plotted as a function of $\theta / \pi$ for a range of values of $B$ and $V$, and the film thickness at $\theta=0, h(0)$, plotted as a function of $B$ for a 


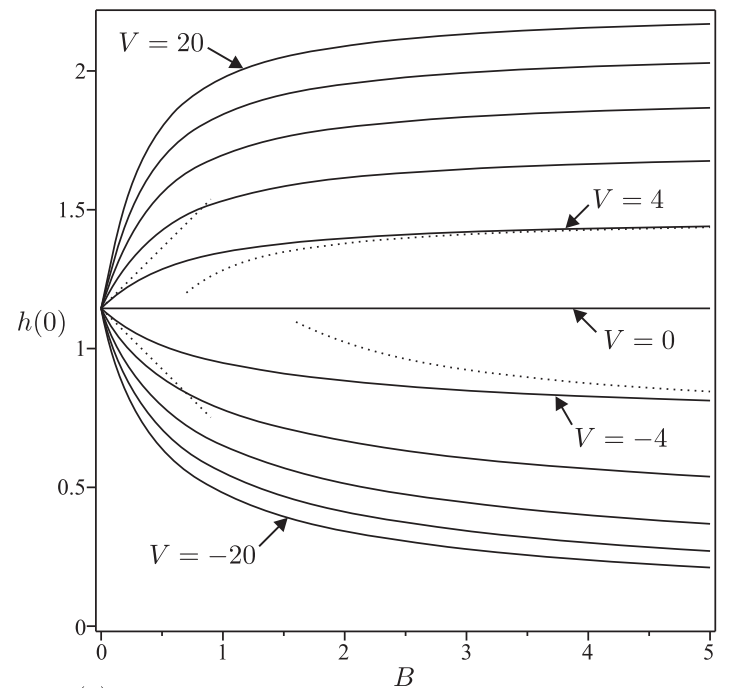

(a)

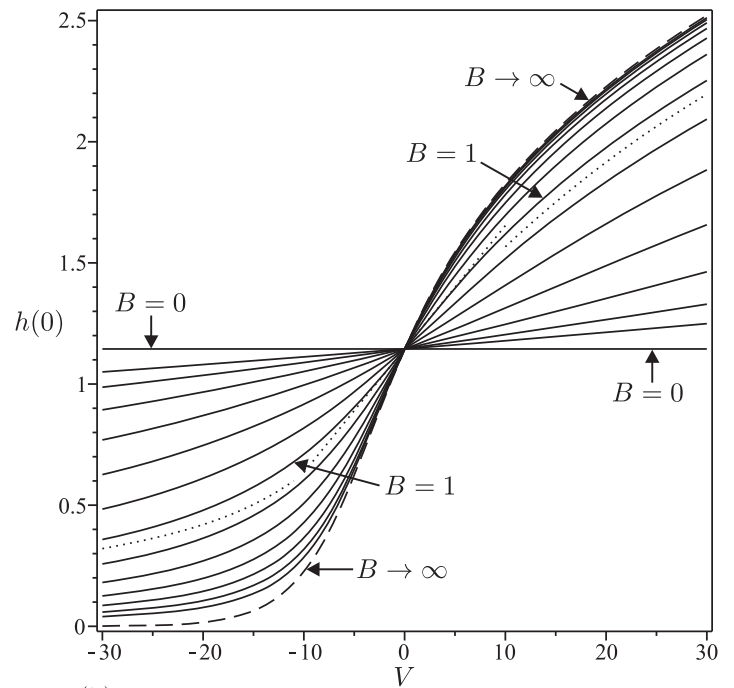

(b)

FIG. 3: Film thickness at $\theta=0, h(0)$, plotted as a function (a) of $B$ for $V=-20,-16,-12, \ldots, 20$ (solid lines) together with the asymptotic solutions in the limits $B \rightarrow 0^{+}$and $B \rightarrow \infty$ in the cases $V=-4$ and 4 (dotted lines), and (b) of $V$ for $B=0$ and $B=10^{n}(n=-1.5,-1.25,-1, \ldots, 1.5)$ (solid lines) together with the asymptotic solutions in the limits $V \rightarrow 0, V \rightarrow \infty$ and $V \rightarrow-\infty$ in the case $B=1$ (dotted lines) and the leading order asymptotic solution in the limit $B \rightarrow \infty$ (dashed line), when $Q=-1 / 2$.

range of values of $V$ and of $V$ for a range of values of $B$, respectively. In particular, Figures 2 and 3 illustrate that $h$ is a monotonically increasing (decreasing) function of $B$ for positive (negative) $V$, and a monotonically increasing function of $V$. In addition, Figure 3 shows good agreement with the asymptotic results for $h$ obtained subsequently.

Figure 4 shows the free-surface temperature at $\theta=0, T(1,0)$, plotted as a function of $B$ for a range of values of $V$ and of $V$ for a range of values of $B$. Taken together with the results for $h$ shown in Figures 2 and 3, Figure 4 illustrates that the free-surface temperature, $T(1, \theta)$, is a monotonically decreasing function of both $B$ and $V$.

Figure 5 shows the velocity $u$ plotted as a function of $Y=h y$ for a range of values of $\theta$ for both a negative and a positive value of $V$, and Figure 6 shows the free-surface velocity at $\theta=0, u(1,0)$, plotted as a function of $B$ for a range of values of $V$ and of $V$ for a range of values of $B$. In particular, Figure 5 shows that the velocity profiles for non-zero values of $V$ are, in general, quite different from the familiar semi-parabolic profile (25) in the constant viscosity case $V=0$. Figure 7 shows typical streamlines of the flow on the right-hand side 

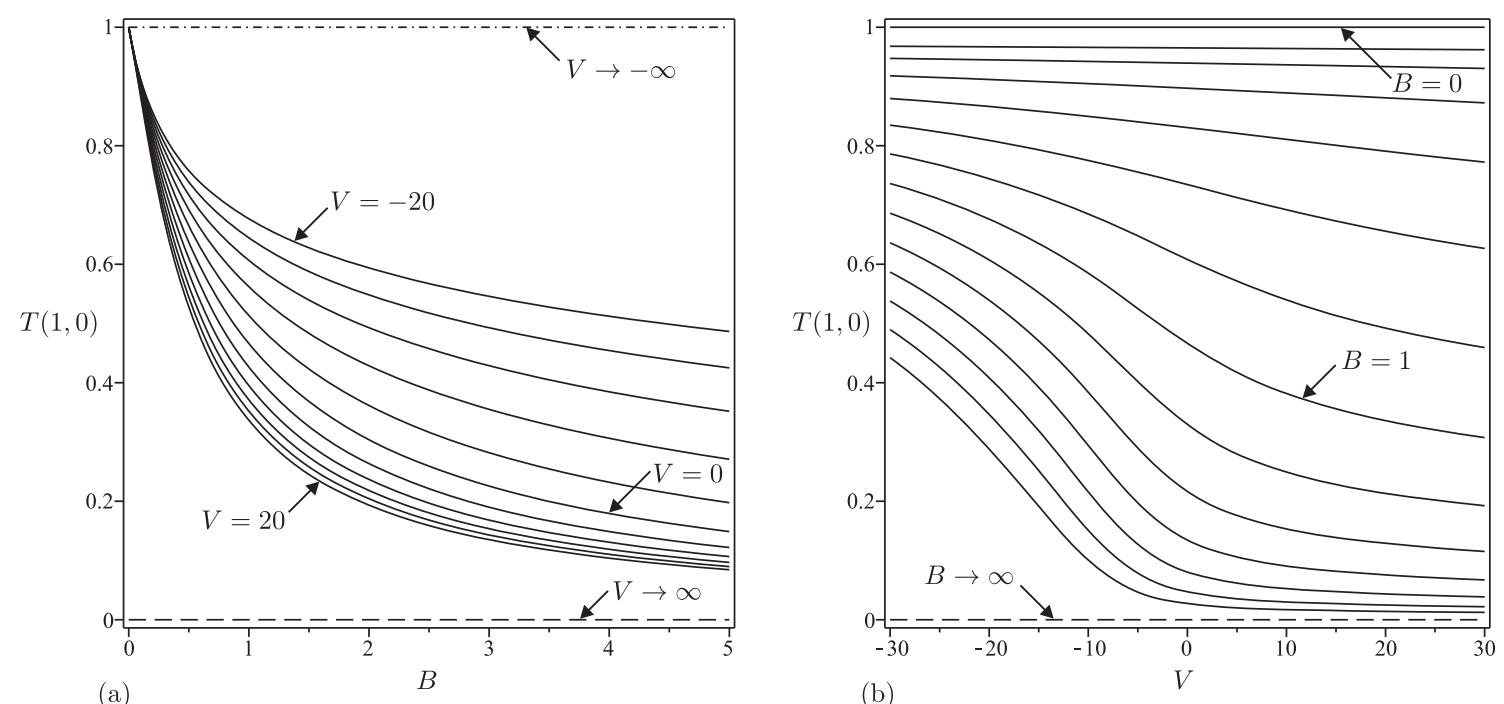

FIG. 4: Free-surface temperature at $\theta=0, T(1,0)$, plotted as a function (a) of $B$ for $V=-20,-16,-12$,

$\ldots, 20$ (solid lines) together with the leading order asymptotic solution in the limit $V \rightarrow \infty$ (i.e.

$T(1,0)=0)($ dashed line) and the leading order asymptotic solution in the limit $V \rightarrow-\infty$ (i.e. $T(1,0)=1$ )

(dash-dotted line), and (b) of $V$ for $B=0$ and $B=10^{n}(n=-1.5,-1.25,-1, \ldots, 1.5)$ (solid lines) together with the leading order asymptotic solution in the limit $B \rightarrow \infty$ (i.e. $T(1,0)=0)$ (dashed line), when $Q=-1 / 2$.

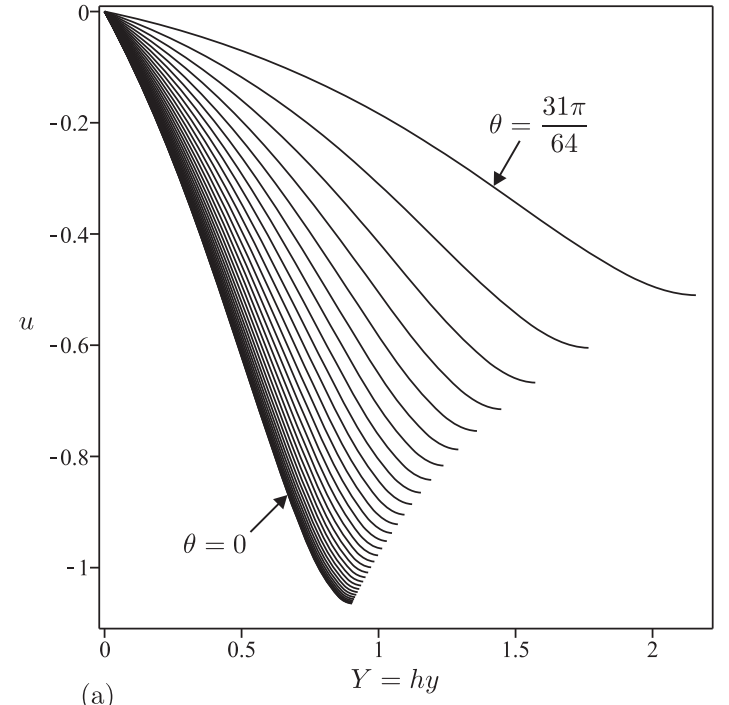

(a)

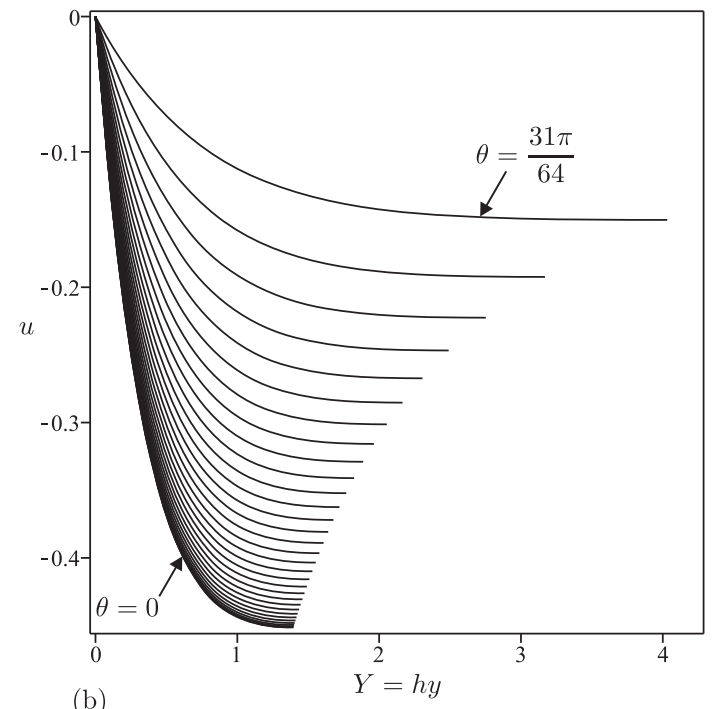

(b)

FIG. 5: Velocity $u$ plotted as a function of $Y=h y$ for $\theta=0, \pi / 64, \pi / 32, \ldots, 31 \pi / 64$ in the cases (a) $V=-5$ and (b) $V=5$, when $Q=-1 / 2$ and $B=1$.

of the cylinder.

Figure 8 shows the load $M$ plotted as a function of $B$ for a range of values of $V$ and of $V$ for a range of values of $B$. In particular, Figure 8 shows that $M$ is a monotonically increasing 


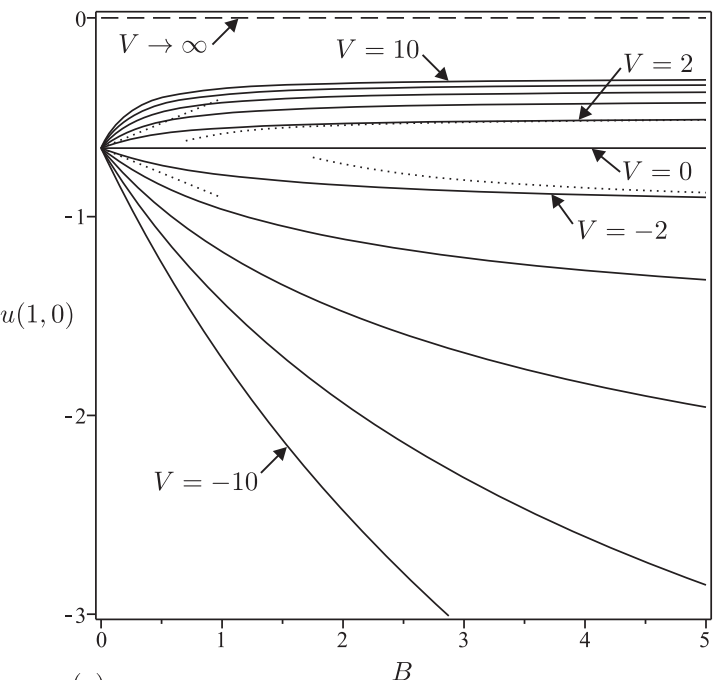

(a)

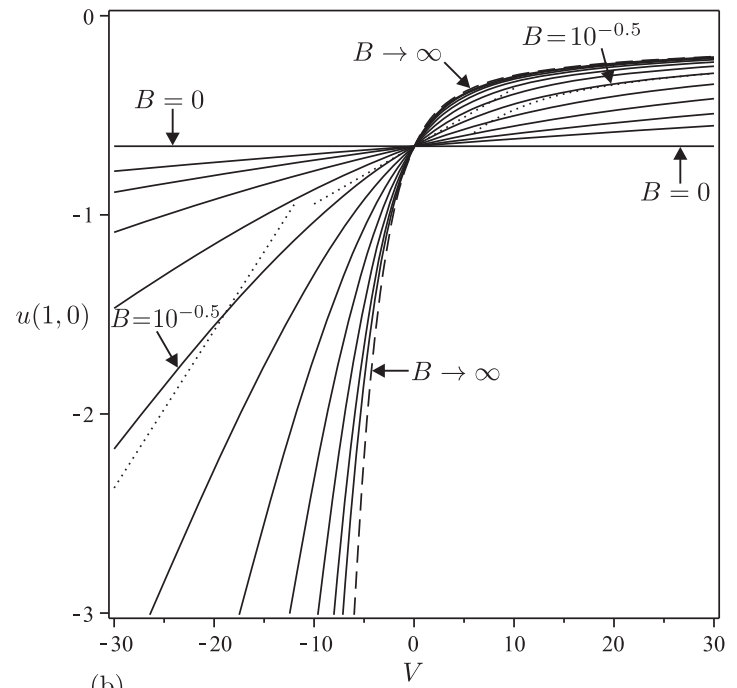

(b)

FIG. 6: Free-surface velocity at $\theta=0, u(1,0)$, plotted as a function (a) of $B$ for $V=-10,-8,-6, \ldots, 10$ (solid lines) together with the asymptotic solutions in the limits $B \rightarrow 0^{+}$and $B \rightarrow \infty$ in the cases $V=-2$ and 2 (dotted lines) and the leading order asymptotic solution in the limit $V \rightarrow \infty$ (i.e. $u(1,0)=0$ ) (dashed line), and (b) of $V$ for $B=0$ and $B=10^{n}(n=-1.5,-1.25,-1, \ldots, 1)$ (solid lines) together with the asymptotic solutions in the limits $V \rightarrow 0, V \rightarrow \infty$ and $V \rightarrow-\infty$ in the case $B=10^{-0.5} \simeq 0.3162$ (dotted

lines) and the leading order asymptotic solution in the limit $B \rightarrow \infty$ (dashed line), when $Q=-1 / 2$.

(decreasing) function of $B$ for positive (negative) $V$, and a monotonically increasing function of $V$. In addition, Figure 8 shows good agreement with the asymptotic results for $M$ obtained subsequently.

In order to obtain a complete understanding of the influence of varying $B$ and $V$, in the following Subsections VA-VE we analyse the behaviour in the asymptotic limits of weak heat transfer at the free surface, $B \rightarrow 0^{+}$, strong heat transfer at the free surface, $B \rightarrow \infty$, weak thermoviscosity, $V \rightarrow 0$, strong positive thermoviscosity, $V \rightarrow \infty$, and strong negative thermoviscosity, $V \rightarrow-\infty$, respectively. In addition, in the Appendix we analyse the distinguished limit of strong thermoviscosity and weak heat transfer, $|V| \rightarrow \infty$ and $B \rightarrow 0^{+}$with $B V=O(1)$, in which, although the variation in temperature across the fluid film small, thermoviscosity effects still enter the problem at leading order. 


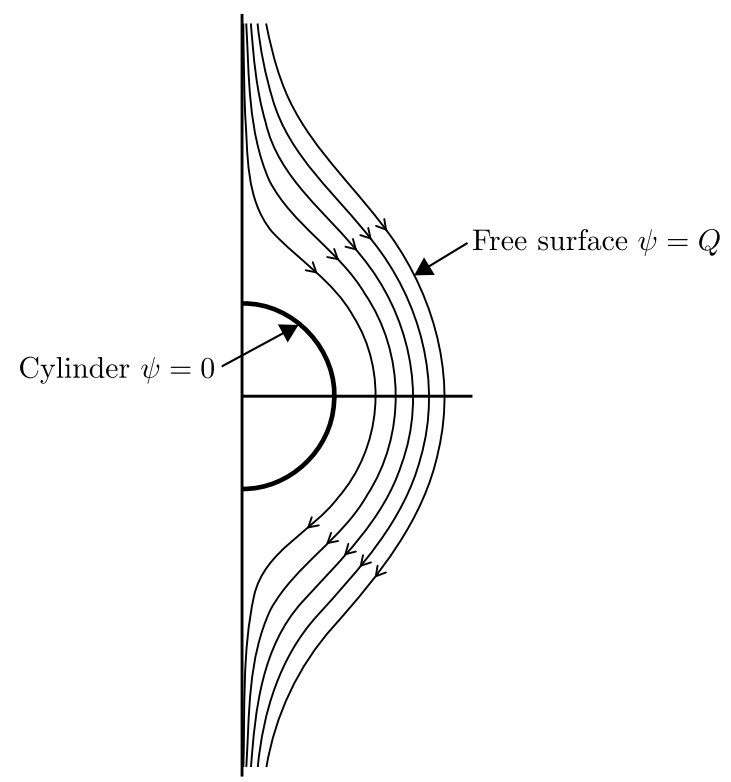

FIG. 7: Typical streamlines of the flow on the right-hand side of the cylinder plotted for $\psi=0$ (the cylinder), $Q / 5,2 Q / 5,3 Q / 5,4 Q / 5$ and $Q$ (the free surface) when $Q=-1 / 2, B=1$ and $V=1$.
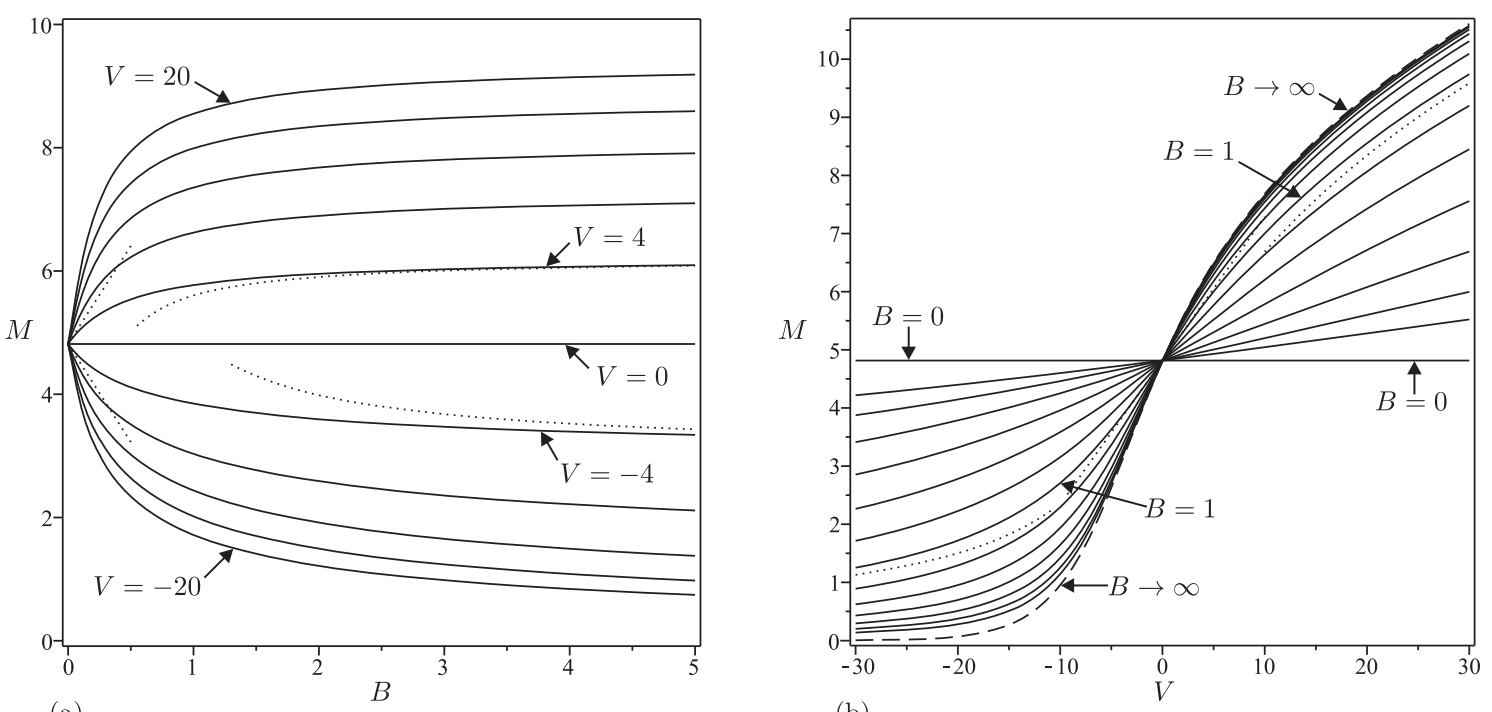

(b)

FIG. 8: Load $M$ plotted as a function (a) of $B$ for $V=-20,-16,-12, \ldots, 20$ (solid lines) together with the asymptotic solutions in the limits $B \rightarrow 0^{+}$and $B \rightarrow \infty$ in the cases $V=-4$ and 4 (dotted lines), and (b) of $V$ for $B=0$ and $B=10^{n}(n=-1.5,-1.25,-1, \ldots, 1.5)$ (solid lines) together with the asymptotic solutions in the limits $V \rightarrow 0, V \rightarrow \infty$ and $V \rightarrow-\infty$ in the case $B=1$ (dotted lines) and the leading order asymptotic solution in the limit $B \rightarrow \infty$ (dashed line), when $Q=-1 / 2$. 


\section{A. The limit of weak heat transfer $B \rightarrow 0^{+}$}

At leading order in the limit of weak heat transfer at the free surface, $B \rightarrow 0^{+}$, the free surface is insulated (i.e. $T_{y}=0$ at $y=1$ ) and, as already discussed in Section IV, the fluid film is isothermal with constant temperature $T \equiv 1$, viscosity $\mu \equiv 1$ and fluidity $f \equiv 1$. Hence the leading-order solutions for $h, u$ and $M$ are simply the isothermal solutions $h_{0}, u_{0}$ and $M_{0}$ given by (24), (25) and (28), respectively.

The effect of variations in $B$ first appear at $O(B)$, to which order the solutions for $h, T$, $u$ and $M$ are given by

$$
\begin{gathered}
h=h_{0}+\frac{B V h_{0}^{2}}{12}+O\left(B^{2}\right), \\
T=1-B h_{0} y+\frac{B^{2}(12-V) h_{0}^{2} y}{12}+O\left(B^{3}\right), \\
u=u_{0}-\frac{B V h_{0}^{3} \cos \theta}{12}\left(4 y^{2}-7 y+2\right) y+O\left(B^{2}\right)
\end{gathered}
$$

and

$$
M=M_{0}+C_{1} Q^{2 / 3} B V+O\left(B^{2}\right)
$$

where the numerical coefficient $C_{1}$ is given by

$$
C_{1}=\frac{1}{6} \int_{0}^{\pi / 2}\left(\frac{3}{\cos \theta}\right)^{2 / 3} \mathrm{~d} \theta=\frac{\pi^{2}}{2^{1 / 3} 3^{5 / 6} \Gamma\left(\frac{2}{3}\right)^{3}}=\frac{C_{0}}{4 \times 3^{1 / 6}} \simeq 1.2629 .
$$

Note that the solutions (38)-(41) are valid for a general viscosity model satisfying $\mu=1$ and $\mathrm{d} \mu / \mathrm{d} T=-V$ when $T=1$ to the order shown (but not to higher orders). The solution (39) shows that the effect of weak heat transfer at the free surface is to decrease $T$ slightly from its constant isothermal value $T \equiv 1$ throughout the fluid film. Thus for positive (negative) thermoviscosity $V>0(V<0)$ the viscosity is slightly increased (decreased) from its constant isothermal value $\mu \equiv 1$, and determining the sign of $u_{1}$ shows that the magnitude of the velocity is slightly increased (decreased) from its value in the isothermal case when $0<y<(7-\sqrt{17}) / 8 \simeq 0.3596$ and slightly decreased (increased) when $(7-\sqrt{17}) / 8<y \leq 1$, with the net effect that the magnitude of the average fluid velocity is slightly decreased 
(increased) and hence that the film thickness (and hence the load) is slightly increased (decreased) everywhere in order to accommodate the fixed volume flux of fluid.

\section{B. The limit of strong heat transfer $B \rightarrow \infty$}

At leading order in the limit of strong heat transfer at the free surface, $B \rightarrow \infty$, the free surface is at the same uniform temperature as the atmosphere (i.e. $T=0$ at $y=1$ ) and the fluid film has non-constant temperature $T=\hat{T}=1-y$ and viscosity $\mu=\hat{\mu}=\mu(\hat{T})$. As Duffy and Wilson ${ }^{45}$ showed, the leading-order solutions for $u$ and $f$, denoted by $\hat{u}$ and $\hat{f}$, are given by

$$
\hat{u}=-\hat{h}^{2} \cos \theta \int_{\hat{T}}^{1} \frac{T}{\mu(T)} d T
$$

and (21), respectively, where $\hat{h}$ denotes the leading-order solution for $h$. Closed-form expressions for $\hat{f}$ for linear, exponential and Eyring viscosity models are described in detail by Wilson and Duffy ${ }^{25}$. Since $\hat{f}$ is a constant (and not a function of $\theta$ as, in general, $f$ is) the leading-order solutions for $h$ and $M$, the latter denoted by $\hat{M}$, are simply given by

$$
\hat{h}=\frac{h_{0}}{\hat{f}^{1 / 3}}=\left(-\frac{3 Q}{\hat{f} \cos \theta}\right)^{1 / 3}, \quad \hat{M}=\frac{M_{0}}{\hat{f}^{1 / 3}}=C_{0}\left(\frac{|Q|}{\hat{f}}\right)^{1 / 3},
$$

where $h_{0}$ and $M_{0}$ are the solutions for $h$ and $M$ of the corresponding isothermal problem with the same flux given by (24) and (28) in which the constant $C_{0}$ is again given by (29). Thus, rather remarkably, for a general viscosity model the film thickness and the load (but not the temperature or the velocity) at leading order in the limit of strong heat transfer are simply re-scaled versions of their values for the corresponding isothermal problem with the same flux. In particular, this means that for positive (negative) thermoviscosity the leading-order film thickness and load are increased (decreased) from their values for the corresponding isothermal problem with the same flux. Furthermore, in this limit $\mathcal{V} \sim V$, and so the leading order expressions for $\mu, u, \psi$ and $f$ are simply given by (30), (32), (33) and (34) with $\mathcal{V}$ replaced by $V$, respectively. 
Note that the re-scaling (44) differs from that proposed by Duffy and Wilson ${ }^{45}$. In general, the leading-order solutions for $h, Q$ and $M$ in the limit of strong heat transfer, denoted by $\hat{h}$, $\hat{Q}$ and $\hat{M}$, are given simply by $\hat{h}=\hat{f}^{-m} h_{0}, \hat{Q}=\hat{f}^{1-3 m} Q_{0}$, and $\hat{M}=\hat{f}^{-m} M_{0}$ for any non-zero value of $m$, where $h_{0}$ and $M_{0}$ are the solutions for $h$ and $M$ of the corresponding isothermal problem with flux $Q_{0}$. Thus at leading order in the limit of strong heat transfer, the film thickness and the load are simply re-scaled versions of their values for the corresponding isothermal problem with the appropriate flux. The present scaling (44) corresponds to the choice $m=1 / 3$ and is simply the special case in which the flux remains unscaled. The scaling proposed by Duffy and Wilson ${ }^{45}$ corresponds to the choice $m=1 / 2$, who showed that this the only possible choice for the corresponding problem of non-isothermal flow on a uniformly rotating cylinder at leading order in the limit of strong heat transfer, but failed to notice that there is no restriction on the value of $m$ for the present problem of non-isothermal flow on a stationary cylinder.

As might have been anticipated, this simple re-scaling property does not extend to higher orders. Specifically, extending the analysis to $O\left(1 / B^{2}\right)$ the solutions for $h, T, u$ and $M$ are given by

$$
\begin{gathered}
h=\hat{h}-\frac{(V+3) \hat{f}-3}{3 \hat{f} B}+O\left(\frac{1}{B^{2}}\right), \\
T=\hat{T}+\frac{y}{B \hat{h}}+\frac{(V \hat{f}-3) y}{3 \hat{f} B^{3} \hat{h}^{2}}+O\left(\frac{1}{B^{3}}\right), \\
u=\hat{u}-\frac{\hat{h} \cos \theta}{3 \hat{f} B V}\left[\frac{6(V-1)}{V}-(2 V+1) \hat{f}\right. \\
\left.+\left(\frac{6[1-V(1-y)]}{V}+[1+V(1-y)(2-3 y)] \hat{f}\right) \exp (-V y)\right]+O\left(\frac{1}{B^{2}}\right)
\end{gathered}
$$

and

$$
M=\hat{M}-\frac{\pi[(V+3) \hat{f}-3]}{3 \hat{f} B}+O\left(\frac{1}{B^{2}}\right) .
$$

The solution (46) shows that the effect of large-but-finite heat transfer at the free surface is to increase $T$ slightly from its leading-order value $T=\hat{T}=1-y$ throughout the fluid film. 

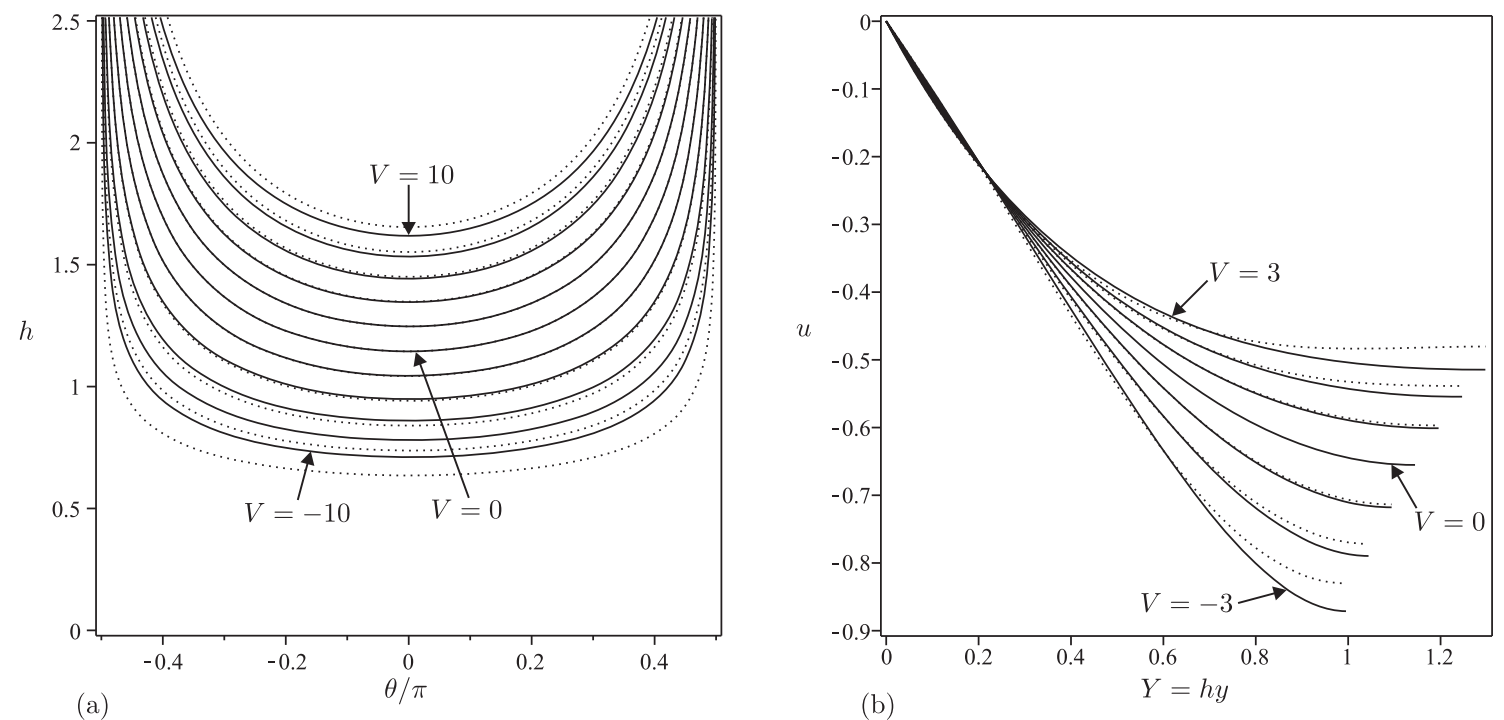

FIG. 9: (a) Film thickness $h$ plotted as a function of $\theta / \pi$ for $V=-10,-8,-6, \ldots, 10$ (solid lines), and (b) velocity $u$ at $\theta=0$ plotted as a function of $Y=h y$ for $V=-3,-2,-1, \ldots, 3$ (solid lines), together with the corresponding asymptotic solutions in the limit $V \rightarrow 0$ (dotted lines), when $Q=-1 / 2$ and $B=1$.

Thus for positive (negative) thermoviscosity $V>0(V<0)$ the viscosity is slightly decreased (increased) from its leading-order value $\mu=\hat{\mu}$ with the net effect that the film thickness is slightly decreased (increased) uniformly in order to accommodate the fixed volume flux of fluid, and hence that the load is slightly decreased (increased).

\section{The limit of weak thermoviscosity $V \rightarrow 0$}

As already discussed in Section IV, at leading order in the limit of weak thermoviscosity, $V \rightarrow 0$, the fluid film has non-constant temperature $T \not \equiv 1$ but constant viscosity $\mu \equiv 1$ and fluidity $f \equiv 1$. From (16) and (17) the solution for $h$ is given by

$$
h=h_{0}+\frac{B V h_{0}^{2}}{12\left(1+B h_{0}\right)}+O\left(V^{2}\right)
$$

and hence from (11), (12) and (18) the solutions for $T, u$ and $M$ are given by

$$
\begin{gathered}
T=1-\frac{B h_{0} y}{1+B h_{0}}-\frac{B^{2} V h_{0}^{2} y}{12\left(1+B h_{0}\right)^{3}}+O\left(V^{2}\right), \\
u=u_{0}-\frac{B V h_{0}^{3} \cos \theta}{12\left(1+B h_{0}\right)}\left(4 y^{2}-7 y+2\right) y+O\left(V^{2}\right)
\end{gathered}
$$



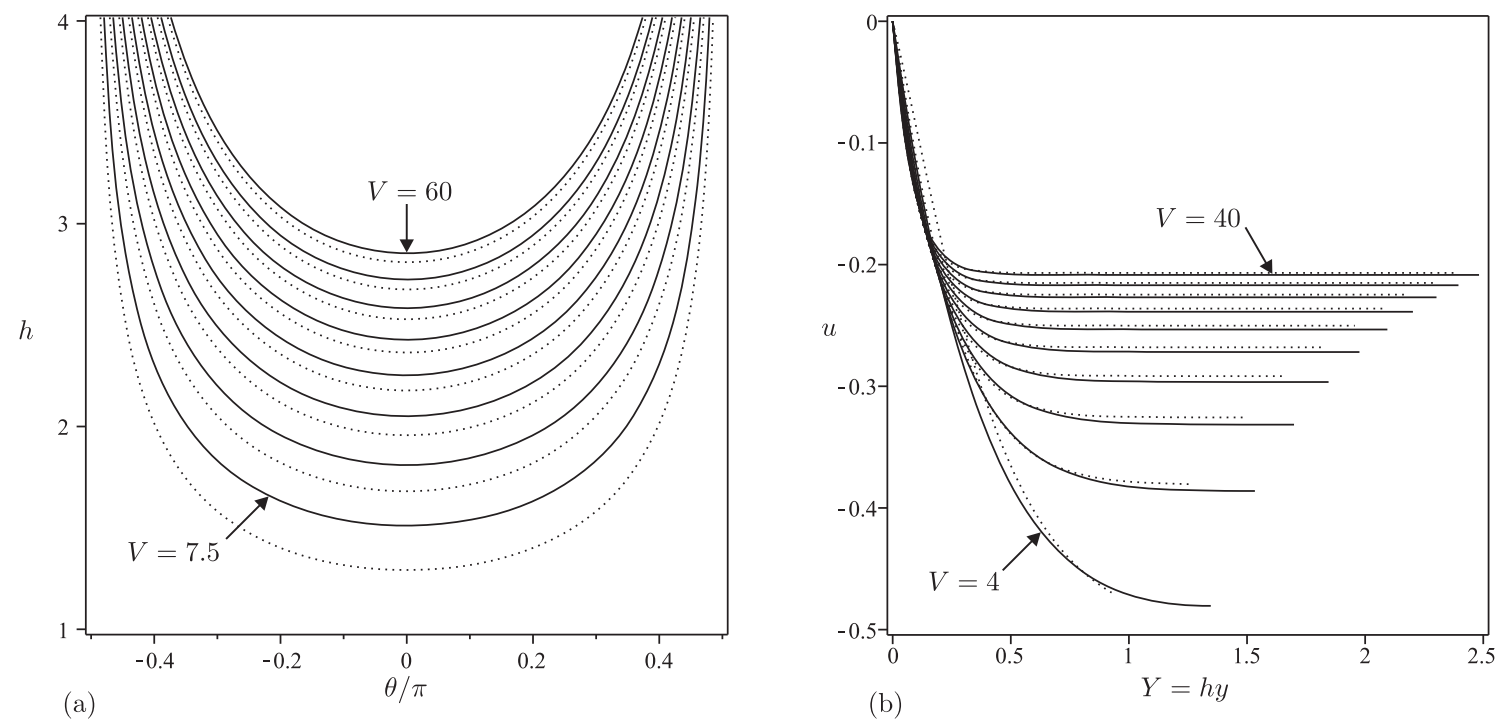

FIG. 10: (a) Film thickness $h$ plotted as a function of $\theta / \pi$ for $V=7.5,15,22.5, \ldots, 60$ (solid lines), and (b) velocity $u$ at $\theta=0$ plotted as a function of $Y=h y$ for $V=4,8,12, \ldots, 40$ (solid lines), together with the corresponding asymptotic solutions in the limit $V \rightarrow \infty$ (dotted lines), when $Q=-1 / 2$ and $B=1$.

and

$$
M=M_{0}+\frac{Q^{2 / 3} B V}{2 \times 3^{1 / 3}} \int_{0}^{\pi / 2} \frac{\mathrm{d} \theta}{(\cos \theta)^{2 / 3}+B(3|Q| \cos \theta)^{1 / 3}}+O\left(V^{2}\right),
$$

where $h_{0}, u_{0}$ and $M_{0}$ are the isothermal solutions given by (24), (25) and (28), respectively. Note that the solutions (49)-(52) are valid for a general viscosity model satisfying $\mu=1$ and $\mathrm{d} \mu / \mathrm{d} T=-V$ when $T=1$ to the order shown (but not to higher orders). The solutions in this limit are similar to those in the limit $B \rightarrow 0^{+}$described in Subsection V A and have a similar physical interpretation. This behaviour is illustrated in Figure 9 which shows the film thickness $h$ plotted as a function of $\theta / \pi$ and the velocity $u$ at $\theta=0$ plotted as a function of $Y=h y$ for a range of values of $V$ near $V=0$.

\section{The limit of strong positive thermoviscosity $V \rightarrow \infty$}

In the limit of strong positive thermoviscosity, $V \rightarrow \infty$, from (16) and (17) the solution for $h$ is given by

$$
h=h_{0}\left(\frac{V}{3}\right)^{1 / 3}-\frac{1}{3 B}+O\left(\frac{1}{V^{1 / 3}}\right)
$$


and hence from (11), (12) and (18) the solutions for $T, u$ and $M$ are given by

$$
\begin{gathered}
T=1-y+\frac{y}{B h_{0}}\left(\frac{3}{V}\right)^{1 / 3}-\frac{2 y}{3 B^{2} h_{0}^{2}}\left(\frac{3}{V}\right)^{2 / 3}+O\left(\frac{1}{V}\right), \\
u=-\frac{h_{0}^{2} \cos \theta}{3}\left(\frac{3}{V}\right)^{1 / 3}[1-\exp (-V y)] \\
-\frac{h_{0} \cos \theta}{9 B}\left(\frac{3}{V}\right)^{2 / 3}[1-(1+3 V y) \exp (-V y)]+O\left(\frac{1}{V}\right)
\end{gathered}
$$

and

$$
M=M_{0}\left(\frac{V}{3}\right)^{1 / 3}-\frac{\pi}{3 B}+O\left(\frac{1}{V^{1 / 3}}\right),
$$

where $h_{0}$ and $M_{0}$ are the isothermal solutions for $h$ and $M$ given by (24) and (28), respectively. In particular, these solutions show that at leading order in the limit of strong positive thermoviscosity the temperature is given by $T=1-y$ and the viscosity $\mu=\exp (V y)$ is exponentially large outside a narrow boundary layer of width $O(1 / V) \ll 1$ near the cylinder $y=0$, resulting in a slow "plug flow" with a uniform (i.e. independent of $y$ ) velocity of $O\left(V^{-1 / 3}\right) \ll 1$ outside the boundary layer and a large film thickness of $O\left(V^{1 / 3}\right) \gg 1$. This behaviour is illustrated in Figure 10 which shows the film thickness $h$ plotted as a function of $\theta / \pi$ and the velocity $u$ at $\theta=0$ plotted as a function of $Y=h y$ for a range of positive values of $V$.

\section{E. The limit of strong negative thermoviscosity $V \rightarrow-\infty$}

In the limit of strong negative thermoviscosity, $V \rightarrow-\infty$, from (16) and (17) the solution for $h$ is given by

$$
h=\frac{1}{B(-V)} \log \left(\frac{Q B^{3} V^{3}}{2 \cos \theta}\right)+O\left(\frac{\log (-V)^{2}}{V^{2}}\right),
$$

and hence from (11), (12) and (18) the solutions for $T, u$ and $M$ are given by

$$
T=1-\frac{y}{(-V)} \log \left(\frac{Q B^{3} V^{3}}{2 \cos \theta}\right)+O\left(\frac{\log (-V)}{V^{2}}\right)
$$




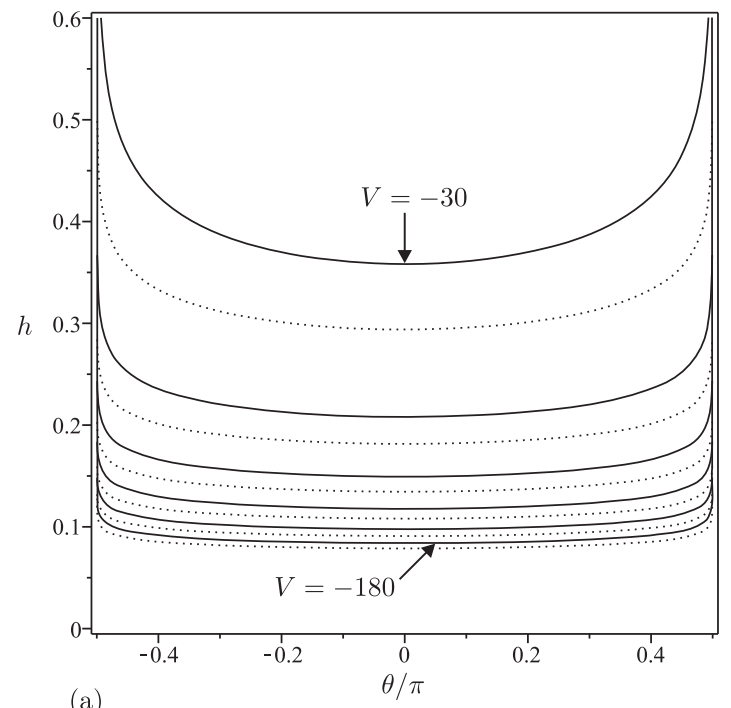

(a)

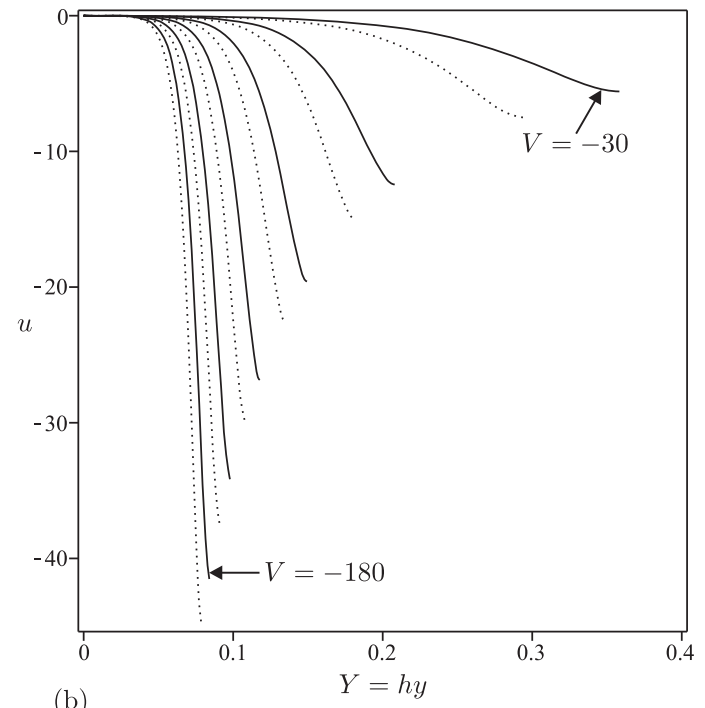

(b)

FIG. 11: (a) Film thickness $h$ plotted as a function of $\theta / \pi$ for $V=-30,-60,-90, \ldots,-180$ (solid lines), and (b) velocity $u$ at $\theta=0$ plotted as a function of $Y=h y$ also for $V=-30,-60,-90, \ldots,-180$ (solid lines), together with the corresponding asymptotic solutions in the limit $V \rightarrow-\infty$ (dotted lines), when $Q=-1 / 2$ and $B=1$.

$$
\begin{gathered}
u=-\frac{\cos \theta}{B^{2} V^{2}}\left[\left(\frac{Q B^{3} V^{3}}{2 \cos \theta}\right)^{y}\left\{\log \left(\frac{Q B^{3} V^{3}}{2 \cos \theta}\right)(1-y)+1\right\}-\log \left(\frac{Q B^{3} V^{3}}{2 \cos \theta}\right)-1\right] \\
+O(\log (-V))
\end{gathered}
$$

and

$$
M=\frac{\pi \log \left(|Q| B^{3}(-V)^{3}\right)}{B(-V)}+O\left(\frac{\log (-V)^{2}}{V^{2}}\right) .
$$

In particular, these solutions show that at leading order in the limit of strong negative thermoviscosity the temperature is given by $T=1$ and the viscosity

$$
\mu=\left(\frac{2 \cos \theta}{Q B^{3} V^{3}}\right)^{y}
$$

decreases from $O(1)$ at the cylinder $y=0$ to $O\left((-V)^{-3}\right) \ll 1$ at the free surface $y=1$ and that the velocity increases from zero at the cylinder (where there is a narrow boundary layer of width $O(1 / \log (-V)) \ll 1)$ to $O(-V) \gg 1$ at the free surface (where there is another narrow boundary layer also of width $O(1 / \log (-V)) \ll 1)$, resulting in a small film thickness of $O(\log (-V) /(-V)) \ll 1$. This behaviour is illustrated in Figure 11 which shows the film thickness $h$ plotted as a function of $\theta / \pi$ and the velocity $u$ at $\theta=0$ plotted as a function of $Y=h y$ for a range of negative values of $V$. 


\section{CONCLUSIONS}

In the present work we obtained a comprehensive description of the two-dimensional steady gravity-driven flow with prescribed volume flux of a thin film of Newtonian fluid with temperature-dependent viscosity on a heated or cooled stationary horizontal cylinder. In particular, we showed that for the exponential viscosity model (7) the effect of increasing $B$ depends on the sign of $V$. When the cylinder is hotter than the surrounding atmosphere (i.e. when $V>0$ ) the effect of increasing $B$ is to decrease the average temperature and so to increase the average viscosity and hence reduce the average velocity within the film, with the net effect that the film thickness (and hence the total fluid load on the cylinder) is increased to maintain the fixed volume flux of fluid. When the cylinder is colder than the surrounding atmosphere (i.e. when $V<0$ ) the opposite occurs. Similarly, we showed that the effect of increasing $V$ is always to increase the film thickness and hence the load. In order to obtain a complete understanding of the influence of varying $B$ and $V$, we also analysed the behaviour in the asymptotic limits of weak heat transfer, $B \rightarrow 0^{+}$, strong heat transfer, $B \rightarrow \infty$, weak thermoviscosity, $V \rightarrow 0$, strong positive thermoviscosity, $V \rightarrow \infty$, and strong negative thermoviscosity, $V \rightarrow-\infty$, as well as (in the Appendix) in the distinguished limit of strong thermoviscosity and weak heat transfer, $|V| \rightarrow \infty$ and $B \rightarrow 0^{+}$with $B V=O(1)$. The asymptotic analysis in the limits $B \rightarrow 0^{+}$and $B \rightarrow \infty$ revealed that increasing $B$ from zero to infinity changes the film thickness everywhere (and hence the load, but not the

temperature or the velocity) by a constant factor of $\hat{f}^{-1 / 3}$, where $\hat{f}$ is given by (21), which depends only on the specific viscosity model considered. The asymptotic analysis in the limits $V \rightarrow 0, V \rightarrow \infty$ and $V \rightarrow-\infty$ revealed that for the exponential viscosity model (7) the behaviour of the solution for large positive thermoviscosity is very different from that for large negative thermoviscosity, and that both are very different from that in the constant viscosity case $V=0$. Specifically, in the limit $V \rightarrow \infty$ the viscosity is exponentially large of $O(\exp (V)) \gg 1$ and the velocity is small and uniform (i.e. independent of $y$ ) of $O\left(V^{-1 / 3}\right) \ll 1$ 
outside a narrow boundary layer of width $O(1 / V) \ll 1$ near the cylinder, leading to a large film thickness of $O\left(V^{1 / 3}\right) \gg 1$, while in the limit $V \rightarrow-\infty$ the viscosity decreases from $O(1)$ at the cylinder to $O\left((-V)^{-3}\right) \ll 1$ at the free surface and the velocity increases from zero at the cylinder to a large value of $O(-V) \gg 1$ at the free surface, leading to a small film thickness of $O(\log (-V) /(-V)) \ll 1$.

\section{APPENDIX A: DISTINGUISHED LIMIT OF STRONG THERMOVISCOSITY AND WEAK HEAT TRANSFER $|V| \rightarrow \infty$ AND $B \rightarrow 0^{+}$WITH $\hat{V}=B V=O(1)$}

Another interesting case also worth considering is the distinguished limit discussed by Wilson and Duffy ${ }^{26}$ of strong thermoviscosity, $|V| \rightarrow \infty$, and weak heat transfer at the free surface, $B \rightarrow 0^{+}$, such that $\hat{V}=B V=O(1)$, in which, although the variation in temperature across the fluid film is small, specifically $T=1-B h y+O\left(B^{2}\right)$, thermoviscosity effects still enter the problem at leading order, i.e. the variation in viscosity across the fluid film is still $O(1)$. Note that in this limit the effective thermoviscosity number, $\hat{V}=B V$, defined in terms of dimensional quantities by

$$
\hat{V}=\frac{\lambda\left(T_{0}-T_{\infty}\right) \epsilon a \alpha_{\mathrm{th}}}{\mu_{0} k_{\mathrm{th}}}
$$

and not the previously defined thermoviscosity number, $V$, is the appropriate nondimensional measure of thermoviscosity effects. In the particular case of the exponential viscosity model $(30)$ in this limit $\mathcal{V} \sim \hat{V} h$ and so the leading order expressions for $\mu, u, \psi$ and $f$ are simply given by (30), (32)-(34) with $\mathcal{V}$ replaced by $\hat{V} h$, respectively.

Note that, in an analogous way to being able to remove $B$ explicitly from the general mathematical problem by rescaling appropriately (discussed in Section III), in this case we could remove $\hat{V}$ explicitly from the mathematical problem by rescaling $h, u, \psi$ and $Q$ appropriately; however, since this again obscures the physical interpretation of the results obtained we retain $\hat{V}$ explicitly in what follows.

In the limit of "weak" thermoviscosity, $\hat{V} \rightarrow 0$, the solutions for $h, u$ and $M$ are given by 
the corresponding results in the limit $B \rightarrow 0$ given in Subsection V A, namely (38), (40) and (41), with $B V$ replaced by $\hat{V}$, and hence have the same physical interpretation.

In the limit of strong positive thermoviscosity, $\hat{V} \rightarrow \infty$, the solutions for $h, u$ and $M$ are given by

$$
\begin{gathered}
h=\left(-\frac{Q \hat{V}}{\cos \theta}\right)^{1 / 2}+\frac{1}{\hat{V}}+O\left(\frac{1}{\hat{V}^{5 / 2}}\right) \\
u=-\frac{\cos \theta}{\hat{V}}\left(-\frac{Q \hat{V}}{\cos \theta}\right)^{1 / 2}\left\{1-\exp \left[-\left(-\frac{Q \hat{V}^{3}}{\cos \theta}\right)^{1 / 2} y\right]+O\left(\frac{1}{\hat{V}^{2}}\right)\right.
\end{gathered}
$$

and

$$
M=\hat{C}(|Q| \hat{V})^{1 / 2}+\frac{\pi}{\hat{V}}+O\left(\frac{1}{\hat{V}^{5 / 2}}\right)
$$

in which the numerical coefficient $\hat{C}$ is given by

$$
\hat{C}=2 \int_{0}^{\pi / 2}\left(\frac{1}{\cos \theta}\right)^{1 / 2} \mathrm{~d} \theta=2 \sqrt{2} \mathrm{~K}\left(\frac{1}{\sqrt{2}}\right) \simeq 5.2441,
$$

where $\mathrm{K}(k)$ is the complete elliptic integral of the first kind with modulus $k$ defined by

$$
\mathrm{K}(k)=\int_{0}^{1} \frac{\mathrm{d} x}{\sqrt{1-x^{2}} \sqrt{1-k^{2} x^{2}}}
$$

(see, for example, Gradshteyn and Ryzhik ${ }^{46}$ ). These solutions differ from the corresponding results in the limit $V \rightarrow \infty$ given in Subsection VD, namely (53), (55) and (56), but have a qualitatively similar physical interpretation. In particular, these solutions show that at leading order in the limit of strong positive thermoviscosity the viscosity

$$
\mu=\exp \left[\left(-\frac{Q \hat{V}^{3}}{\cos \theta}\right)^{1 / 2} y\right]
$$

is exponentially large outside a narrow boundary layer of width $O\left(\hat{V}^{-3 / 2}\right) \ll 1$ near the cylinder $y=0$, resulting in a slow "plug flow" with a uniform (i.e. independent of $y$ ) velocity of $O\left(\hat{V}^{-1 / 2}\right) \ll 1$ outside the boundary layer and a large film thickness of $O\left(\hat{V}^{1 / 2}\right) \gg 1$.

In the limit of strong negative thermoviscosity, $\hat{V} \rightarrow-\infty$, the solutions for $h, u$ and $M$ are given by

$$
h=\frac{1}{(-\hat{V})} \log \left(\frac{Q \hat{V}^{3}}{2 \cos \theta}\right)+O\left(\frac{\log \left((-\hat{V})^{6}\right)}{\hat{V}^{4}}\right)
$$




$$
\begin{gathered}
u=-\frac{\cos \theta}{\hat{V}^{2}}\left[\left(\frac{Q \hat{V}^{3}}{2 \cos \theta}\right)^{y}\left\{\log \left(\frac{Q \hat{V}^{3}}{2 \cos \theta}\right)(1-y)+1\right\}-\log \left(\frac{Q \hat{V}^{3}}{2 \cos \theta}\right)-1\right] \\
+O\left(\frac{\log \left((-\hat{V})^{6}\right)}{\hat{V}^{2}}\right)
\end{gathered}
$$

and

$$
M=\frac{\pi \log \left(|Q|(-\hat{V})^{3}\right)}{(-\hat{V})}+O\left(\frac{\log \left((-\hat{V})^{6}\right)}{\hat{V}^{4}}\right) .
$$

At leading (but not higher) order these solutions coincide with the corresponding results in the limit $V \rightarrow-\infty$ given in Subsection VE, namely (57), (59) and (60), and hence have the same physical interpretation.

\section{ACKNOWLEDGEMENTS}

The first author (GAL) gratefully acknowledges the financial support of the United Kingdom Engineering and Physical Sciences Research Council (EPSRC) via a Doctoral Training Account (DTA) research studentship. This work was completed while the second author (SKW) was a Visiting Fellow in the Department of Mechanical and Aerospace Engineering in the School of Engineering and Applied Science at Princeton University, USA.

1 W. Nusselt, "Die Oberflächenkondensation des Wasserdampfes," Z. Vereines deutscher Ingenieure 60, 541-546 (1916).

2 W. Nusselt, "Die Oberflächenkondensation des Wasserdampfes," Z. Vereines deutscher Ingenieure 60, 569-575 (1916).

3 E. M. Sparrow and J. L. Gregg, "Laminar condensation heat transfer on a horizontal cylinder," J. Heat Transfer 81, 291-296 (1959).

4 A. A. Nicol, Z. L. Aidoun, R. J. Gribben, and G. Wilks, "Heat transfer in the presence of condensate drainage," Int. J. Multiphase Flow 14, 349-359 (1988).

5 J.-J. Shu and G. Wilks, "Heat transfer in the flow of a cold, two-dimensional draining sheet over a hot, horizontal cylinder," Eur. J. Mech. B. Fluids 28, 185-190 (2009).

6 I. G. Shekriladze and V. I. Gomelauri, "Theoretical study of laminar film condensation of flowing vapour," Int. J. Heat Mass Transfer 9, 581-591 (1966). 
7 T. Fujii, H. Uehara, and C. Kurata, "Laminar filmwise condensation of flowing vapour on a horizontal cylinder," Int. J. Heat Mass Transfer 15, 235-246 (1972).

8 J. W. Rose, "Effect of pressure gradient in forced convection film condensation on a horizontal tube," Int. J. Heat Mass Transfer 27, 39-47 (1984).

9 C.-K. Chen and Y.-T. Lin, "Laminar film condensation from a downward-flowing steam-air mixture onto a horizontal circular tube," Appl. Math. Modell. 33, 1944-1956 (2009).

10 P. K. Sarma, B. Vijayalakshmi, F. Mayinger, and S. Kakac, "Turbulent film condensation on a horizontal tube with external flow of pure vapors," Int. J. Heat Mass Transfer 41, 537-545 (1998).

11 S.-A. Yang and Y.-T. Lin, "Turbulent film condensation on a non-isothermal horizontal tube effect of eddy diffusivity," Appl. Math. Modell. 29, 1149-1163 (2005).

12 S.-A. Yang and C.-H. Hsu, "Free- and forced-convection film condensation from a horizontal elliptic tube with a vertical plate and a horizontal [sic] tube as special cases," Int. J. Heat Fluid Flow 18, 567-574 (1997).

13 Y.-T. Lin and S.-A. Yang, "Turbulent film condensation on a horizontal elliptical tube," Heat Mass Transfer 41, 495-502 (2005).

14 B. Reisfeld and S. G. Bankoff, "Non-isothermal flow of a liquid film on a horizontal cylinder," J. Fluid Mech. 236, 167-196 (1992).

15 A. T. Conlisk and J. Mao, "Nonisothermal absorption on a horizontal cylindrical tube -1 . The film flow," Chem. Eng. Sci. 51, 1275-1285 (1996).

16 D. Goussis and R. E. Kelly, "Effects of viscosity variation on the stability of film flow down heated or cooled inclined surfaces: Long-wavelength analysis," Phys. Fluids 28, 3207-3214 (1985).

17 D. A. Goussis and R. E. Kelly, "Effects of viscosity variation on the stability of a liquid film flow down heated or cooled inclined surfaces: Finite wavelength analysis," Phys. Fluids 30, 974-982 (1987).

18 C.-C. Hwang and C.-I. Weng, "Non-linear stability analysis of film flow down a heated or cooled inclined plane with viscosity variation," Int. J. Heat Mass Transfer 31, 1775-1784 (1988).

19 B. Reisfeld and S. G. Bankoff, "Nonlinear stability of a heated thin liquid film with variable viscosity," Phys. Fluids A 2, 2066-2067 (1990).

20 M.-C. Wu and C.-C. Hwang, "Nonlinear theory of film rupture with viscosity variation," Int. Comm. Heat Mass Transfer 18, 705-713 (1991).

21 R. Selak and G. Lebon, "Bénard-Marangoni thermoconvective instability in presence of a temperature-dependent viscosity," J. Phys. II France 3, 1185-1199 (1993).

22 D. Bercovici, "A theoretical model of cooling viscous gravity currents with temperaturedependent viscosity," Geophys. Res. Lett. 21, 1177-1180 (1994).

23 N. J. Balmforth and R. V. Craster, "Dynamics of cooling domes of viscoplastic fluid," J. Fluid Mech. 422, 225-248 (2000).

24 Yu. O. Kabova and V. V. Kuznetsov, "Downward flow of a nonisothermal thin liquid film with variable viscosity," J. Appl. Mech. Tech. Phys. 43, 895-901 (2002). 
25 S. K. Wilson and B. R. Duffy, "On the gravity-driven draining of a rivulet of fluid with temperature-dependent viscosity down a uniformly heated or cooled substrate," J. Eng. Math. 42, 359-372 (2002).

26 S. K. Wilson and B. R. Duffy, "Strong temperature-dependent-viscosity effects on a rivulet draining down a uniformly heated or cooled slowly varying substrate," Phys. Fluids 15, 827-840 (2003).

27 B. R. Duffy and S. K. Wilson, "A rivulet of perfectly wetting fluid with temperature-dependent viscosity draining down a uniformly heated or cooled slowly varying substrate," Phys. Fluids 15, 3236-3239 (2003).

28 A. Sansom, J. R. King, and D. S. Riley, "Degenerate-diffusion models for the spreading of thin non-isothermal gravity currents," J. Eng. Math. 48, 43-68 (2004).

29 R. Usha, R. Ravindran, and B. Uma, "Dynamics and stability of a thin liquid film on a heated rotating disk film with variable viscosity," Phys. Fluids 17, 102103 (2005).

30 L. Wu, "Spin coating of thin liquid films on an axisymmetrically heated disk," Phys. Fluids 18, $063602(2006)$.

31 C.-K. Lin, C.-C. Hwang, and T.-C. Ke, "Three-dimensional nonlinear rupture theory of thin liquid films on a cylinder," J. Colloid Interface Sci. 256, 480-482 (2002).

32 A. A. King, L. J. Cummings, S. Naire, and O. E. Jensen, "Liquid film dynamics in horizontal and tilted tubes: Dry spots and sliding drops," Phys. Fluids 19, 042102 (2007).

33 L. R. Band, D. S. Riley, P. C. Matthews, J. M. Oliver, and S. L. Waters, "Annular thin-film flows driven by azimuthal variations in interfacial tension," Q. J. Mech. Appl. Math. 62, 403-430 (2009).

34 O. Haimovich and A. Oron, "Nonlinear dynamics of a thin liquid film on an axially oscillating cylindrical surface," Phys. Fluids 22, 032101 (2010).

35 H. K. Moffatt, "Behaviour of a viscous film on the outer surface of a rotating cylinder," J. Méc. 16, 651-673 (1977).

36 V. V. Pukhnachev, "Motion of a liquid film on the surface of a rotating cylinder in a gravitational field,” J. Appl. Mech. Tech. Phys. 18, 344-351 (1977).

37 R. E. Johnson, "Steady-state coating flows inside a rotating horizontal cylinder," J. Fluid Mech. 190, 321-342 (1988).

38 B. R. Duffy and S. K. Wilson, "Thin-film and curtain flows on the outside of a rotating horizontal cylinder," J. Fluid Mech. 394, 29-49 (1999).

39 J. Ashmore, A. E. Hosoi, and H. A. Stone, "The effect of surface tension on rimming flows in a partially filled rotating cylinder," J. Fluid Mech. 479, 65-98 (2003).

40 M. Villegas-Díaz, H. Power, and D. S. Riley, "Analytical and numerical studies of the stability of thin-film rimming flow subject to surface shear," J. Fluid Mech. 541, 317-344 (2005).

41 E. S. Benilov, M. S. Benilov, and N. Kopteva, "Steady rimming flows with surface tension," J. Fluid Mech. 597, 91-118 (2008).

42 P. L. Evans, L. W. Schwartz, and R. V. Roy, "Three-dimensional solutions for coating flow on 
a rotating horizontal cylinder: Theory and experiment," Phys. Fluids 17, 072102 (2005).

43 M. A. Kelmanson, "On inertial effects in the Moffatt-Pukhnachov coating-flow problem," J. Fluid Mech. 633, 327-353 (2009).

44 R. Hunt, "Numerical solution of the free-surface viscous flow on a horizontal rotating elliptical cylinder," Numer. Methods Partial Differential Eq. 24, 1094-1114 (2008).

45 B. R. Duffy and S. K. Wilson, "Large-Biot-number non-isothermal flow of a thin film on a stationary or rotating cylinder," Eur. Phys. J. Special Topics 166, 147-150 (2009).

46 I. S. Gradshteyn and I. M. Ryzhik, Tables of Integrals, Series and Products, 2nd ed., (Academic Press, 1980). pp. 904-925. 